\title{
JUNO sensitivity to low energy atmospheric neutrino spectra
}

\author{
The JUNO Collaboration
}

Received: 10 June 2021 / Accepted: 19 August 2021 / Published online: 8 October 2021

(C) The Author(s) 2021

\begin{abstract}
Atmospheric neutrinos are one of the most relevant natural neutrino sources that can be exploited to infer properties about cosmic rays and neutrino oscillations. The Jiangmen Underground Neutrino Observatory (JUNO) experiment, a 20 kton liquid scintillator detector with excellent energy resolution is currently under construction in China. JUNO will be able to detect several atmospheric neutrinos per day given the large volume. A study on the JUNO detection and reconstruction capabilities of atmospheric $v_{e}$ and $v_{\mu}$ fluxes is presented in this paper. In this study, a sample of atmospheric neutrino Monte Carlo events has been generated, starting from theoretical models, and then processed by the detector simulation. The excellent timing resolution of the 3" PMT light detection system of JUNO detector and the much higher light yield for scintillation over Cherenkov allow to measure the time structure of the scintillation light with very high precision. Since $v_{e}$ and $v_{\mu}$ interactions produce a slightly different light pattern, the different time evolution of light allows to discriminate the flavor of primary neutrinos. A probabilistic unfolding method has been used, in order to infer the primary neutrino energy spectrum from the detector experimental observables. The simulated spectrum has been reconstructed between $100 \mathrm{MeV}$ and $10 \mathrm{GeV}$, showing a great potential of the detector in the atmospheric low energy region.
\end{abstract}

\section{Introduction}

Atmospheric neutrinos are a naturally occurring neutrino source. They originate from the decays of $\pi$ and $K$ produced in extensive air showers initiated by the interaction of cosmic rays with the Earth's atmosphere [1-4]. The energy spectrum of primary cosmic rays above $100 \mathrm{MeV}$ can be described by a power law $\frac{d N}{d E} \propto E^{-\gamma}$, where the spectral index is $\gamma \simeq 2.7$ for $E \leq 10^{6} \mathrm{GeV}$ and $\gamma \simeq 3.0$ above that value [5].

\footnotetext{
ae-mail: Juno_pub_comm@juno.ihep.ac.cn
}

At energies larger than $5 \times 10^{8} \mathrm{GeV}$, the spectrum becomes steeper $(\gamma \simeq 3.2)[6]$ and it flattens again $(\gamma \simeq 2.7)$ when $E \geq 3 \times 10^{9} \mathrm{GeV}$. In the interaction of a single high energy Cosmic Ray with the nuclei of the Earth's atmosphere, hundreds or thousands mesons can be produced. The atmospheric neutrino energy spectrum spans a wide range from the $\mathrm{MeV}$ up to the PeV scale and can be roughly described by a power law [7-11]. The spectral index is, in general, steeper than that of primary cosmic rays, since the parent mesons lose a large fraction of their energy before decaying. The spectrum intensity is suppressed at sub-GeV energies reflecting the rigidity cutoff, that describes the shielding provided by the geomagnetic field against the arrival of cosmic rays particles from outside the magnetosphere. Neutrinos originating from muon decays contribute mainly up to a few GeV. The flavor ratio $\left(v_{\mu}+\bar{v}_{\mu}\right) /\left(v_{e}+\bar{v}_{e}\right)$ is around two at $\sim 1 \mathrm{GeV}$ and increases as the energy increases, since more muons are likely to reach the Earth's surface without decaying. At energies above hundreds of $\mathrm{GeV}$, the decay length of $\pi$ and $K$ becomes longer than their path length in the atmosphere, leading to a neutrino flux reduction. At the highest energies, the decay of heavy charmed mesons is expected to dominate the atmospheric neutrino production. Given the very short lifetime of these particles, the associated neutrino flux is commonly referred as "prompt" [12-14].

Since the Earth is mostly transparent to neutrinos below the PeV energy scale, an atmospheric neutrino detector is able to see neutrinos coming from all directions. The distance from the production point to the detector varies from $O(10)$ to $O\left(10^{4}\right) \mathrm{km}$, depending on the zenith angle [15]. The angular distribution has a characteristic shape with an increased flux towards the horizontal direction (with respect to the vertical direction), due to the longer path length of parent particles in the atmosphere. In the sub-GeV energy region there is an asymmetry along the East-West axis, which reflects the azimuthal dependence of the rigidity cutoff of the cosmic rays.

Atmospheric neutrinos were detected for the first time in the 1960s [16,17]. Further measurements led to the discovery 


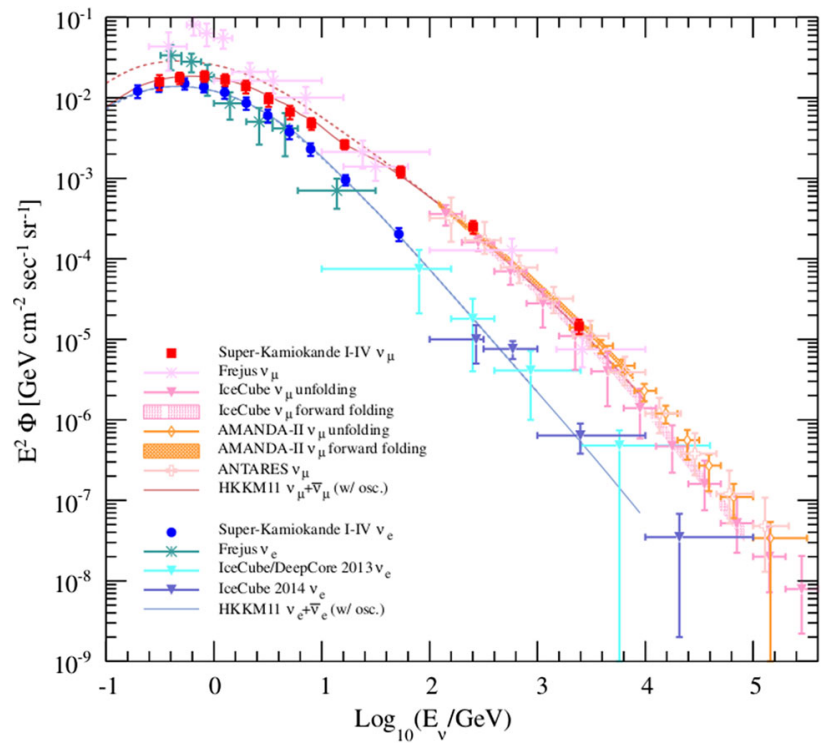

Fig. 1 Present measurements of the atmospheric neutrino energy spectrum, compared with theoretical predictions. Data and models are reported separately for $v_{e}$ and $v_{\mu}$. Figure from [7]

of neutrino flavor oscillations in 1998 [18]. Some of the missing pieces in the puzzle of neutrino physics are going to be addressed also by means of atmospheric neutrinos. The field of research is currently very active and several experiments are scheduled in the coming years to answer the unsolved questions. Next-generation detectors for atmospheric neutrino physics plan to significantly improve performances, compared to present ones, by increasing their size and detection granularity. The efforts are mostly concentrated on flavor oscillation physics, pushing the detectors sensitivity for the neutrino mass ordering (MO) and the CP phase $\delta$ in the neutrino sector. The most prominent examples are DUNE [19], Hyper-Kamiokande [20], INO [21], ORCA [22], and PINGU [23].

In Fig. 1, present measurements of the energy spectrum of atmospheric neutrinos are reported, including predictions from theoretical models.

Measurements performed over the last decades, up to present times, are able to cover a very wide range in the neutrino energy, from several hundreds of $\mathrm{MeV}$ to several hundreds of TeV. This sector has been explored predominantly by Cherenkov detectors, such as Super-Kamiokande [7] and IceCube [8-10,24]. The Jiangmen Underground Neutrino Observatory (JUNO), currently under construction in China, will be able to detect several atmospheric neutrinos per day. JUNO is going to become the largest liquid scintillator (LS) based detector ever built, having a target LS mass more than one order of magnitude larger than present ones. The large detector mass is one of the key-points for atmospheric neutrino detection, since it is comparable to the largest present water-based detector, Super-Kamiokande. Despite the lim- ited ability of JUNO in tracking single particles after a neutrino interaction, with respect to large Cherenkov detectors, and a slightly reduced accessible statistics, the LS nature of the detector allows more precise measurements towards the low energy region. This sector of the energy spectrum is still not fully covered by present and past experiments. Furthermore, it also corresponds to the region where theoretical models have the largest uncertainties.

The atmospheric neutrino flux measurements by means of the JUNO detector allow to investigate the neutrino $\mathrm{MO}$ and the $\theta_{23}$ octant. It is possible to pursue also the $\mathrm{CP}$ phase $\delta$ measurement. In our work, we investigate JUNO's potential for measuring the atmospheric $v_{e}$ and $v_{\mu}$ fluxes in the energy range $100 \mathrm{MeV}-10 \mathrm{GeV}$.

\section{JUNO experiment}

The JUNO experiment $[25,26]$ is a LS neutrino detector currently under construction in a dedicated underground laboratory (about $700 \mathrm{~m}$ deep, 1800 m.w.e.) near Kaiping, Jiangmen city, Guandong province (P. R. China). A sketch of the detector is shown in Fig. 2. The central detector (CD) consists of $20 \mathrm{kton}$ of LS, contained in a $12 \mathrm{~cm}$ thick, highly transparent, acrylic sphere with a diameter of $35.4 \mathrm{~m}$. The light produced in the LS is read out by 17,612 20" high quantum efficiency (QE) photomultiplier tubes (PMTs) and 25,600 3" PMTs, providing a total photo-coverage of more than $75 \%$. About 13,000 of the 20" PMTs are Microchannel Plate (MCP) PMTs, developed by the JUNO collaboration and currently being produced by the North Night Vision Technology company. The remaining 5000 20" PMTs consist of the R12860 model produced by Hamamatsu. Both of these PMTs have a photon detection efficiency greater than $27 \%$. For the 20" PMTs, the full waveform will be acquired. Their large photon collection area, however, has the consequence of a large dark noise rate, on average of the order of $30 \mathrm{kHz}$, and a time resolution on single photo-electrons in the range from 1 to $10 \mathrm{~ns}$. The additional 3" PMTs, built by the HZC company, are deployed in the 20" PMTs' lattice structure, in order to reduce any possible systematics due to the loss of linearity in charge reconstruction and to improve the timing measurements [27]. Due to their small area, the 3" PMTs will operate in digital mode, thus being an independent readout system that can be exploited for cross-calibrating the 20" PMTs energy response. This feature becomes extremely important for high-energy events, where millions of photons are produced. Furthermore, due to the size difference, the Transit Time Spread (TTS) of 3" PMTs is of the order of the nanosecond, while the 20" PMTs one is larger, on average.

The acrylic sphere is surrounded by a stainless steel truss structure, which has a diameter of about $40 \mathrm{~m}$ and constitutes the mechanical support for both the acrylic sphere and the 


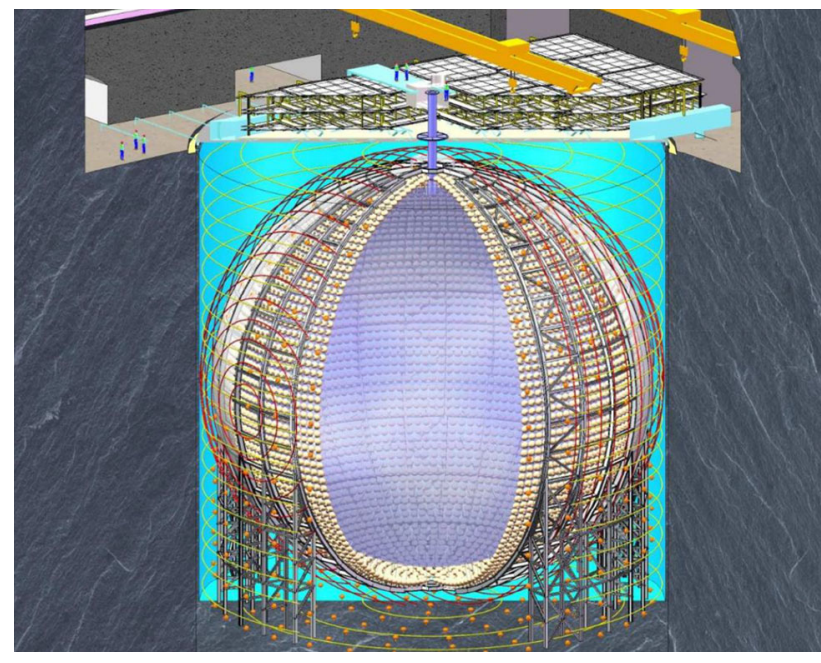

Fig. 2 Layout of the JUNO detector

PMTs. The central detector is submerged in a $\sim 44 \mathrm{~m}$ deep water pool (WP) filled with $\sim 30$ kton of ultrapure water and instrumented with 240020 " MCP-PMTs. It acts as an active Cherenkov muon veto and shields the $\mathrm{CD}$ against external radioactivity. The walls of the pool are covered with highreflectivity Tyvek film, in order to increase the photon collection and allowing to veto Cosmic Ray muons with $>95 \%$ efficiency. A Top Tracker (TT) is placed on top of the water pool, to improve the total veto efficiency and the reconstruction of atmospheric muons. The TT consists of three layers of scintillator strip detectors, refurbished after the decommissioning of the OPERA experiment Target Tracker [28]. It has a granularity of $2.6 \times 2.6 \mathrm{~cm}^{2}$ and a coverage of about $60 \%$ of the WP top surface.

The JUNO LS mixture consists of three components: linear alkylbenzene (LAB) as solvent, $2.5 \mathrm{~g} / \mathrm{l}$ of 2,5diphenyloxazole (PPO) as scintillation fluor and $3 \mathrm{mg} / \mathrm{l}$ of 1,4-Bis(2-methylsyryl) benzene (bis-MSB) as wavelength shifter [29]. This mixture ensures an effective light yield of $\sim 10^{4}$ photons per $\mathrm{MeV}$ of deposited energy and an attenuation length greater than $20 \mathrm{~m}$ for $430 \mathrm{~nm}$ photons. The designed radio-purity levels of the JUNO LS are $\mathcal{O}\left(10^{-16}\right)$ $\mathrm{g} / \mathrm{g}$ for the bulk ${ }^{238} \mathrm{U},{ }^{232} \mathrm{Th}$, and ${ }^{40} \mathrm{~K}$ contaminants [30]. The calibration of the JUNO CD will be performed by four different systems [31]. An automated calibration unit (ACU) will deploy different radioactive sources along the detector vertical axis. The ACU system is also designed to deploy a laser source, with a photon intensity that can cover a range from hundreds of $\mathrm{keV}$ up to $\mathcal{O}(\mathrm{TeV}$ ) equivalent energy. Two Cable Loop Systems (CLS) will instead place sources across two planes. A guide tube (GT) system, installed on the outer circumference of the sphere, will provide information regarding non-uniformity at the $\mathrm{CD}$ boundary. A Remote Operated Vehicle (ROV) will finally deploy sources in the whole detec- tor volume. Periodical calibration campaigns will ensure to keep the overall energy resolution around $3 \% / \sqrt{E / \mathrm{MeV}}$ in the $\mathrm{MeV}$ energy region, where the analysis for the neutrino MO will focus. Atmospheric neutrinos interacting inside JUNO can produce different final states, depending on the nature of the interaction they undergo. A first distinction can be done between charged-current $(\mathrm{CC})$ and neutral-current (NC) interactions. In the first case, the lepton of the same flavor of the interacting neutrino is produced and therefore the original neutrino information is preserved. In NC interactions, on the contrary, only a hadronic state is visible and the flavor of the interacting neutrino cannot be inferred. In the energy of interest for atmospheric neutrinos, the dominant interaction is the neutrino-nucleon scattering. The most prominent channels are the elastic and quasi-elastic scattering, the resonant production, and the deep inelastic scattering [32]. This last classification concerns only about the final hadronic products, which give information about the original energy of the neutrino, but are not sensitive to the interaction flavor. Instead, the $\mathrm{CC}$ or the $\mathrm{NC}$ nature of the neutrino interaction implies a fundamental difference in the visible products. Apart from the flavor information, the absence of the flavor-corresponding lepton in the final state for NC interactions means also that the neutrino carries away part of its initial energy, which is not released inside the detector. NC events are therefore expected to be concentrated at lower values of the visible energy, while $\mathrm{CC}$ ones dominate at higher energy.

\section{Monte Carlo dataset}

The study of the atmospheric neutrino flux is usually based on the predictions of the expected flux made by Monte Carlo simulations. In this work, we consider the predictions from the latest version of the HKKM model [33], which we refer to as HKKM14 hereafter. The model assumes a Cosmic Ray spectrum based on BESS [34,35] and AMS-01 [36] measurements. The DPMJET-III [37] and the JAM [38] hadronic interaction models are used for the simulation of the interaction with the Earth's atmosphere. The HKKM model provides the calculation of the expected atmospheric neutrino flux at different locations, taking into account the latitude and longitude of the detector. The energy range spans from $100 \mathrm{MeV}$ up to $10 \mathrm{TeV}$. Solar modulation and the asymmetry in the azimuthal distribution are also considered. In the HKKM parametrization, the neutrino flux is calculated at the source and therefore no oscillation effects are included. The HKKM14 atmospheric neutrino flux prediction, calculated at the JUNO experimental site, is shown in Fig. 3. Hereafter, $v_{e}$ and $v_{\mu}$ will be used to label both neutrinos and antineutrinos of electron and muon flavor, respectively. 


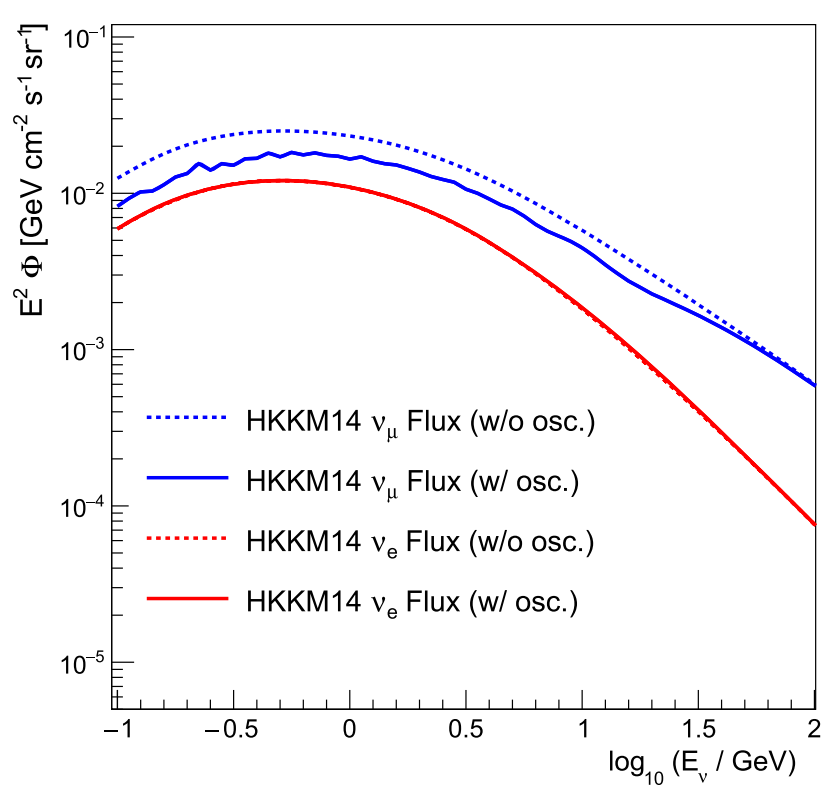

Fig. 3 Expected atmospheric (neutrino + antineutrino) flux at the JUNO site, for $v_{e}$ (red) and $v_{\mu}$ (blue), according to the HKKM14 model [33]. The flux is reported with (full line) and without (dashed line) considering neutrino oscillations

In order to get a realistic prediction, neutrino oscillations have been applied to the original flux, including matter effects. The impact of the oscillation effects has been evaluated according to the standard 3-neutrinos mixing scheme [39].

The interaction of atmospheric neutrinos with the JUNO detector has been simulated by means of the GENIE Neutrino Monte Carlo Generator [40,41] inside an energy range up to $20 \mathrm{GeV}$. The elemental composition of the neutrino target has been set as the one of the JUNO LS (mainly ${ }^{12} \mathrm{C}$ and ${ }^{1} \mathrm{H}$, with relative composition of 0.88 and 0.12 , respectively). The output of the simulation contains information about the type of interaction that neutrinos undergo, either a $\mathrm{CC}$ or a $\mathrm{NC}$ one, and the full list of secondary particles and their associated properties (Particle ID, momentum, direction, .... ). The contribution of $v_{\tau}$ CC interactions has been found not to affect the analysis results by independent evaluations and have been therefore not considered in the present work. Secondary particles produced in the interaction between neutrinos and the JUNO target material have been propagated in the detector by using a GEANT4-based Monte Carlo simulation. The JUNO detector simulation code has been developed within the SNi PER framework [42]. In the detector simulation, several physical processes are included: electromagnetic interaction, decay, hadronic elastic and inelastic interactions, scintillation (including re-emission), Cherenkov emission, and optical absorption. A detailed optical model, including the optical properties of all the detector materials, is also implemented. The output relevant for the analysis includes the timestamp, the number of photoelectrons, and the position of each PMT hit. A data sample of about $5 \times 10^{5} v_{\mu}+v_{e}$ events have been generated, hereafter called large data sample, in order to set up the procedure used to reconstruct the atmospheric neutrino spectrum and to understand the detector response over a large statistics of events. A sample of 6500 events have been injected in the simulation as a separate Monte Carlo data sample, corresponding to a detector live time of about 5 years. This smaller data sample is hereafter identified as small data sample.

\section{Analysis strategy}

As a large LS detector, JUNO achieves its best performance on events which are fully-contained within the volume, where a calorimetric measurement can be performed. Partiallycontained events, having some secondaries escaping from the $\mathrm{CD}$ active volume, are reconstructed with a worse energy resolution. This analysis therefore targets fully-contained events, to be accounted for reconstruction. This sets an intrinsic upper limit of $\sim 10 \mathrm{GeV}$ on the $v_{\mu}$ flux, since the highenergy muons produced in a CC interaction always escape the CD volume. For $v_{e}\left(v_{\mu}\right)$, the "golden" events consist of $v_{e}\left(v_{\mu}\right)$ fully-contained and CC events and the components to reduce are partially-contained and $\mathrm{NC}$ events of all flavor neutrinos. Through-going muons, that may be produced in $v_{\mu}$ interactions with materials surrounding the $\mathrm{CD}$, are not considered in this study.

\subsection{Fiducial cuts}

Before applying the analysis selection to isolate $v_{e}$ and $v_{\mu}$ populations, some preliminary cuts are applied to the large neutrino sample, with the aim of removing low-quality events. A first cut on the interaction vertex position is applied, in order to remove events which release their energy near the edge of the acrylic sphere. These events typically exhibit a loss of linearity between the deposited and the collected energy, because part of the energy is released in the acrylic and water and not in the LS and because the closest PMTs collect a great amount of light and can undergo saturation. A Gaussian smearing with $\sigma=1 \mathrm{~m}$ has been applied to the MC interaction point (hereafter called vertex), in order to reproduce the uncertainty on the reconstructed position. We require that $R_{\text {vertex }}$ (i.e. the distance between the vertex and the center of the detector) is less than $16 \mathrm{~m}$ to ensure a linear detector response. The precision in the reconstruction vertex at lower energy (in the $\mathrm{MeV}$ range) is in general much better than $1 \mathrm{~m}$; on the contrary, at the $\mathrm{GeV}$ energy scale, secondary particles can deposit their energy on a long track and the events can no longer be considered as point-like. It has been checked that even an error of few meters on the ver- 
tex position does not affect the performance of the selection procedure.

As described in Sect. 2, the CD is surrounded by a water Cherenkov detector acting as veto for atmospheric muons. Both muons and secondaries coming from partiallycontained neutrino events can release a certain amount of energy in the WP and produce a large amount of Cherenkov photons. Therefore, in order to remove partially-contained neutrino events and suppress the atmospheric muon background, we require the total number of hits seen by the water pool veto PMTs $\left(N_{\text {hits }}^{W P}\right)$ to be less than 50, including the contribution from PMT dark noise. This latter term can become important for WP PMTs, because the single-count rate due to WP PMTs dark noise is high (up to several tens of $\mathrm{kHz}$ ) and the total number of prompt hits from muons Cherenkov light can be small. Hits on WP PMTs are considered in a $200 \mathrm{~ns}$ time window, which is approximately the time needed by a muon to cross the entire detector. The dark hits contribution is simulated on a statistical basis, assuming a binomial distribution. After applying the fiducial cuts described previously, the simulated large neutrino sample is composed at $97 \%$ of fully-contained event. The remaining partiallycontained events are composed at $96 \%$ of $v_{\mu}$ CC interactions. The total efficiency for all $v_{e}$ events is $68 \%$, for $v_{\mu}$ events is $63 \%$.

\subsection{Atmospheric muon background}

The atmospheric muon background consists of the secondary muon flux produced after the interaction of cosmic rays with the atmosphere, in the same way as for neutrinos. The JUNO detector location is about $700 \mathrm{~m}$ underground, therefore part of the muon radiation is able to penetrate the rock overburden and release energy inside the detector. The energy released by atmospheric muons inside JUNO is comparable with that of particles coming from atmospheric neutrino interactions (hundreds of $\mathrm{MeV}$ - several GeV). Muons can mimic the topology of atmospheric neutrino events and can therefore be a source of background. Although the external water Cherenkov veto is designed to reject these events with high efficiency, the atmospheric muon event rate is several orders of magnitude higher than that from atmospheric neutrino interactions. From preliminary calculations, their event rate inside the JUNO CD is around $3-4 \mathrm{~Hz}$, corresponding to roughly $10^{5}$ times the atmospheric neutrino event rate, considering that the average energy of atmospheric muons reaching JUNO is $207 \mathrm{GeV}$. The desired acceptance rate for the atmospheric muon background must be therefore at least of the order of $10^{-5}$. In order to get a comprehensive picture of the atmospheric muon flux within the framework of this study, a full MC simulation is necessary. Atmospheric muons produce several millions of photons in the JUNO LS and the full detector simulation requires high CPU power and stor- age. For this purpose, a sample of only $10^{5}$ muon events has been generated, according to the energy and angular distributions evaluated at the JUNO site. The expected muon flux in the detector is calculated within the JUNO Collaboration according to a parametrized model at Earth surface [4] and simulating muons propagation through matter [43]. A detector simulation has been performed. Atmospheric muons in JUNO appear as high-energy tracks which release a large amount of energy both in the WP and in the CD. The fiducial cuts described in Sect. 4.1 require instead a low collected light inside the WP.

Hereafter, the readout charge of the event, in terms of the number of PEs collected by CD 20" PMTs, is called NPE. NPE represents the observable used to reconstruct the neutrino energy. The same fiducial cuts have been applied to the muon sample, with the additional request of more than $10^{5}$ NPE, which is the region of interest for the analysis. An acceptance of $<2.3 \times 10^{-5}$ at $90 \%$ confidence level is achieved. The accuracy in the estimation of the acceptance will be improved by increasing the Monte Carlo statistics.

\subsection{Neutrino flavor identification}

As mentioned above, $v_{e}\left(v_{\mu}\right) \mathrm{CC}$ interactions are the preferred detection channels, since the corresponding charged leptons have very different behaviours. Electrons lose energy quickly via bremsstrahlung and ionization and even at $\mathrm{GeV}$ energies their track length is no more than 1-2 $\mathrm{m}$. On the contrary, muons with energy greater than $1 \mathrm{GeV}$ have longer tracks inside the detector volume. Low-energy muons, moreover, may decay inside the scintillator volume and give a delayed energy release from the Michel electron. The above differences make $v_{\mu} \mathrm{CC}$ events more extended in time and space, with respect to $v_{e} \mathrm{CC}$ events. The latter component has indeed a much shorter evolution. Hadronic particles are common to all classes of events and make up the visible part of NC events. Hadrons, in general, have a long energy release, because of their interactions and decays.

The event time profile can be therefore exploited to discriminate between different classes of events [44]. A highprecision measurement of the photon arrival time is an important requirement. For this reason, the timing information is taken from the data of the 3" PMT system of the JUNO detector, which have a low TTS value. A Gaussian smearing with a typical width $\sigma=1.6 \mathrm{~ns}$ (taken from preliminary measurements) has been applied to the true Monte Carlo hit time over each 3" PMT. In order to be aligned to a realistic DAQ window, only events inside a $1.2 \mu \mathrm{s}$ time window have been considered. A time residual $t_{\text {res }}$ is then defined for each hit on the i-th 3" PMT as: 

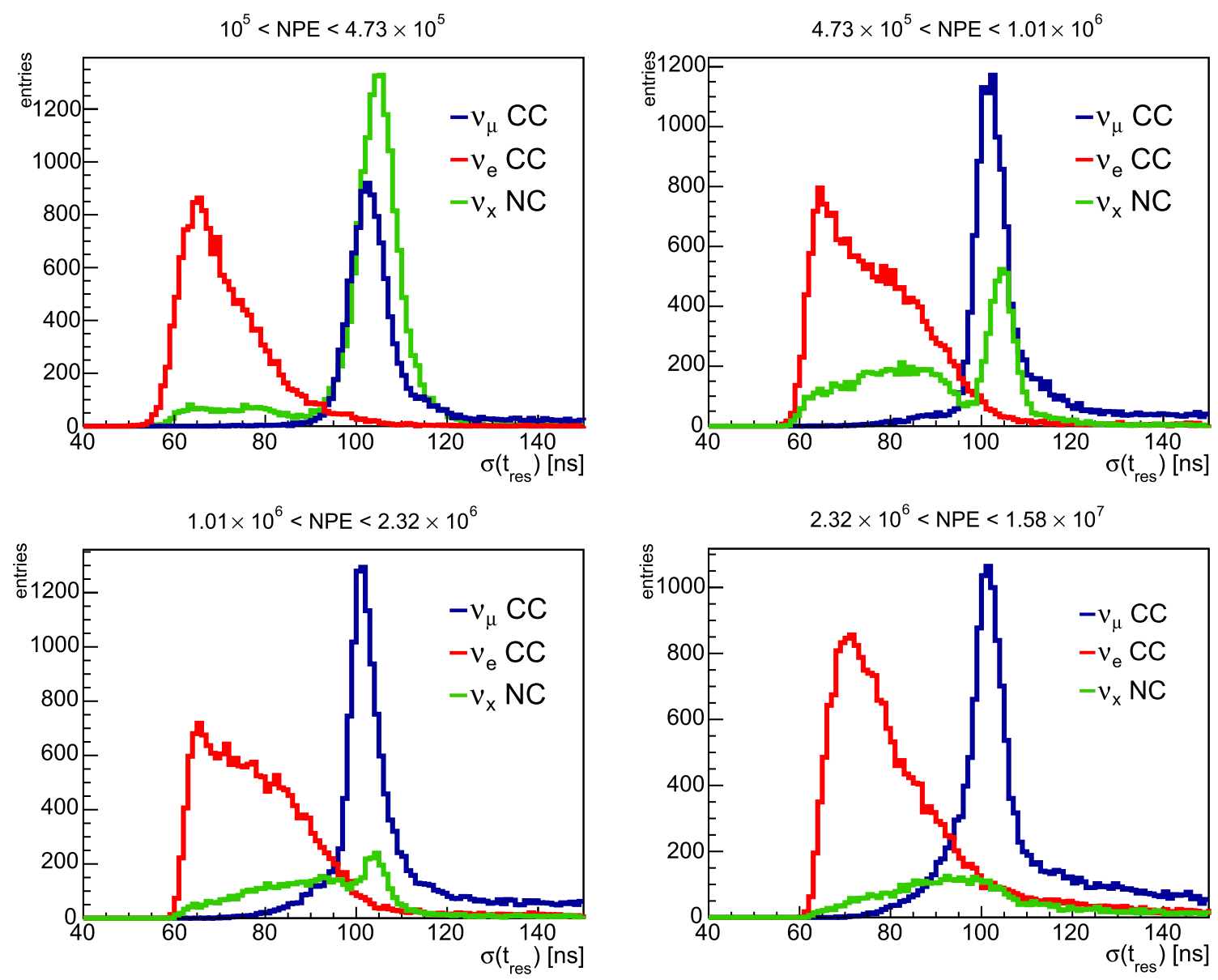

Fig. 4 Distribution of $\sigma\left(t_{r e s}\right)$ for $v_{\mu} \mathrm{CC}$ (blue), $v_{e} \mathrm{CC}$ (red) and NC events (green), for different ranges of NPE

$t_{\text {res }}^{i}=t_{\text {hit }}^{i}-\left(\frac{n \cdot R_{V}^{i}}{c}\right)$,

where $t_{h i t}^{i}$ is the hit time on the i-th 3 " PMT, $n$ is the refraction index of the JUNO liquid scintillator and $R_{V}^{i}$ is the distance between the reconstructed vertex position and the i-th 3 " PMT. The time profile of the scintillation light emitted by $v_{e}$ and $v_{\mu} \mathrm{CC}$ events is different and the latter has a more prominent tail; therefore, the RMS of the $t_{\text {res }}$ distribution hereafter called $\sigma\left(t_{\text {res }}\right)$ - over the fired 3" PMTs can be used as discrimination parameter. In Fig. 4 , the $\sigma\left(t_{\text {res }}\right)$ distribution is reported for the three populations: $v_{\mu} \mathrm{CC}, v_{e} \mathrm{CC}$, and $\mathrm{NC}$ events. The variable is also reported separately in 4 different intervals of NPE, selected such as to have equal statistics in each of them.

The plots in Fig. 4 show a good separation between the $v_{e} \mathrm{CC}$ and the $v_{\mu} \mathrm{CC}$ component, over the whole energy range. The $\mathrm{NC}$ component appears to be overlapped mainly to $v_{\mu} \mathrm{CC}$ events, with a tail also in the $v_{e} \mathrm{CC}$ region. The reason is that a large fraction of the hadronic component of the secondaries is made of pions, that either decay to $v_{\mu}+$ $\mu\left(\pi^{+} / \pi^{-}\right)$or to two gammas $\left(\pi^{0}\right)$. The first category is almost indistinguishable from $v_{\mu} \mathrm{CC}$ events, while the second one results in electromagnetic showers and resembles the $v_{e} \mathrm{CC}$ component. The relative weight of charged and neutral pions in the final state changes across the energy, as well as that of nucleons. This feature is at the origin of the different shape of the $\sigma\left(t_{\text {res }}\right)$ distribution for NC events in Fig. 4, since each of the four bins of NPE corresponds to a different energy interval. Protons and neutrons, moreover, have a time profile similar to the one of muons, because they result in a long-lasting energy release inside the LS. The contribution of NC component, however, becomes less significant at high energy, due to its steeper spectral shape. In NC events, indeed, part of the initial energy of the interacting neutrino is carried away by the neutrino itself and is not deposited inside the detector. Given the different features, two separate selection criteria are used to maximize CC events. In order to separate $v_{e}$ events, a value of $\sigma\left(t_{r e s}\right)<75 \mathrm{~ns}$ is required. The cut results in an efficiency for $v_{e}$ events $\simeq 42 \%$, with respect to the large sample after fiducial cuts, and a residual contamination from $v_{\mu}$ less than $6 \%$. A requirement of $\sigma\left(t_{\text {res }}\right)>95 \mathrm{~ns}$ is required to isolate $v_{\mu}$ events. In order to reduce the contribution from $\mathrm{NC}$ events at low energy, an 

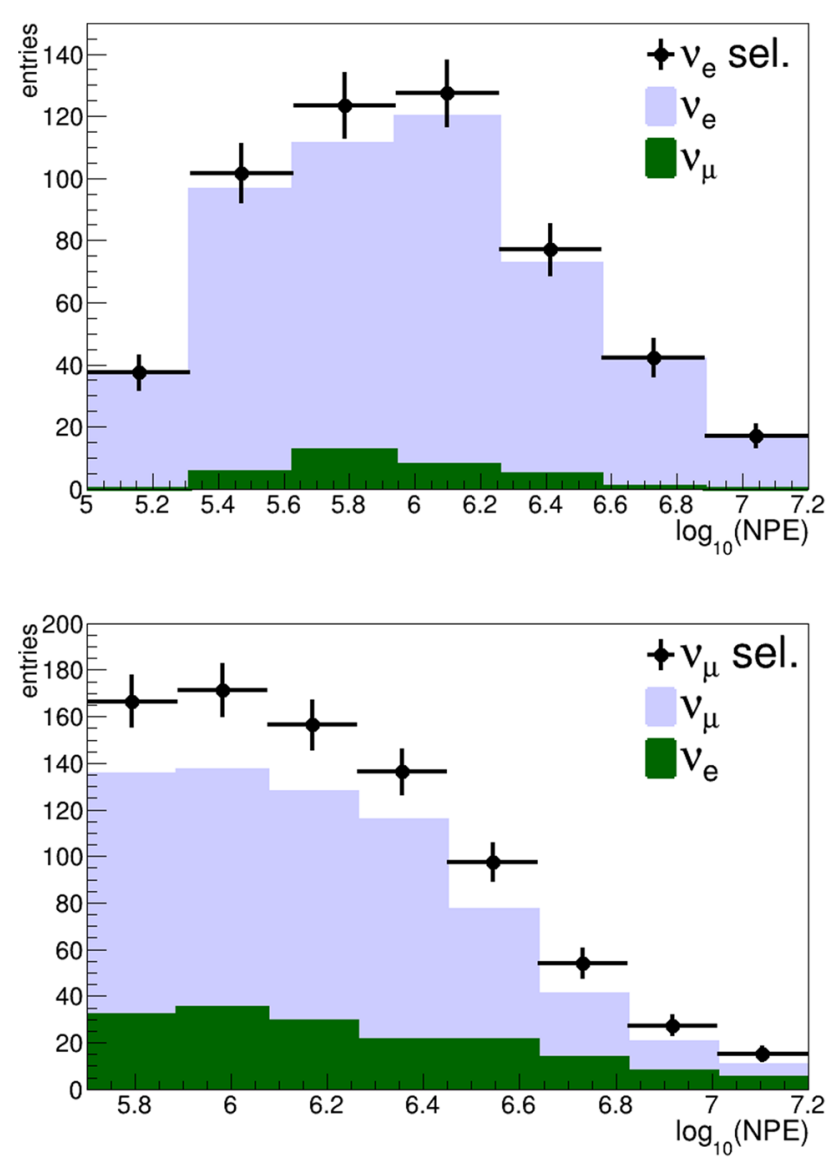

Fig. 5 Distribution of the observable $\log _{10}$ (NPE) in the analysis bins, after the $v_{e}$ selection (top) and the $v_{\mu}$ selection (bottom). The black dots represent the number of selected events in every bin $j$, with associated statistical error. The filled histograms reproduce the bin composition, in terms of the correct flavor (light blue) and wrong flavor (green)

additional requirement of NPE $\geq 5 \times 10^{5}$ has been set for $v_{\mu}$ selection, thus limiting the analysis to events with a neutrino energy $\gtrsim 400 \mathrm{MeV}$. The efficiency for $v_{\mu}$ events is $85 \%$ with respect to the large sample after fiducial cuts and the residual $v_{e}$ contamination is less than $20 \%$. The residual NC events are populated both by $v_{e}$ and $v_{\mu}$.

The flavor identification procedure has been extensively checked by means of an independent analysis. A variation of the $\sigma\left(t_{\text {res }}\right)$ cut has been applied and the resulting efficiency and contamination are in a very good agreement within the statistical and systematic errors reported in this work.

In order to test the JUNO performance in reconstructing the atmospheric neutrino flux, we used the small Monte Carlo sample corresponding to $\sim 5$ years of data-taking described in Sect. 3. The energy range considered for the atmospheric $v_{e}$ flux is $[-1.00,1.05]$, expressed in $\log _{10}\left(E_{v} / \mathrm{GeV}\right)$ units and is divided in seven bins. The corresponding $\log _{10}(\mathrm{NPE})$ range is [5.0, 7.2], divided in seven bins as well. Similarly, the energy range for $v_{\mu}$ is $[-0.30,1.05]$, divided in seven bins, and the corresponding $\log _{10}(\mathrm{NPE})$ range is [5.7, 7.2], divided
Table 1 Summary of selections flow for $v_{e}$ and $v_{\mu}$ fluxes, in terms of number of events in the analysis region, applied to the small data sample corresponding to $\sim 5$ years of data taking. The values are reported before the selections, after the fiducial cuts and after the $\sigma\left(t_{\text {res }}\right)$ selection. The residual background is also reported

\begin{tabular}{lll}
\hline & $v_{e}$ & $v_{\mu}$ \\
\hline Events injected in the simulation & 6500 & \\
Charge region & 1725 & 1241 \\
Fiducial cuts & 1167 & 773 \\
$\sigma\left(t_{\text {res }}\right)$ cut & 495 & 661 \\
Residual background & 30 & 163 \\
\hline
\end{tabular}

in eight bins. The distribution of $\log _{10}(\mathrm{NPE})$ is reported in Fig. 5, in the bins used in the analysis. A summary of the small sample population is in Table 1, as a function of the flavor and of the cuts applied, in the NPE regions considered.

\subsection{Unfolding}

The determination of the atmospheric neutrino energy spectrum, starting from the detector experimental observables, is a classical unfolding problem. In this case, the true spectrum is deconvolved from the distribution of the experimental observables, knowing the detector response. In the classical fitting method, on the other hand, the true distribution is extracted from the observables by directly comparing the experimental distribution with the results of a model prediction. The main benefit of the unfolding is that it does not require a particular choice of the spectrum parametrization. In a liquid scintillator detector like JUNO, the main observable for the energy reconstruction is the total number of photoelectrons NPE detected by the 20" PMTs. This value is related to the total energy deposit in the LS and therefore to the neutrino energy. The neutrino energy spectrum $E_{v}$ is then unfolded from the NPE spectrum. In general, the observable NPE spectrum $N$ can be expressed in terms of the primary neutrino spectrum $E$ as

$N_{j}=\sum_{i} A_{j i} E_{i}$,

where $A_{j i}$ is the likelihood matrix, which can be estimated by means of a full detector simulation. The relationship in Eq. 2 can be inverted by using the unfolding matrix $U_{i j}$ :

$$
E_{i}=\sum_{j} U_{i j} N_{j}
$$

The unfolding matrix $U_{i j}$ can be evaluated by means of an iterative Bayesian procedure [45]. In this case, the likelihood matrix $A_{j i}$ can be expressed as the probability $P\left(N_{j} \mid E_{i}\right)$ of detecting an event in the $j$-th bin of the NPE spectrum $N_{j}$ produced by the interaction of a neutrino in the $i$-th bin of the 
energy spectrum $E_{i}$. The values of $P\left(N_{j} \mid E_{i}\right)$ are evaluated by means of a Monte Carlo detector simulation and normalized as $\sum_{j} A_{j i}=1-\varepsilon_{i}$, where $\varepsilon_{i}$ takes into account the inefficiency in measuring the energy $E_{i}$. The wrong-flavor events are also included in $A_{j i}$. Using Bayes' theorem, the unfolding matrix $U_{i j}$ can be written as:

$U_{i j}=P\left(E_{i} \mid N_{j}\right)=\frac{P\left(N_{j} \mid E_{i}\right) P_{0}\left(E_{i}\right)}{\sum_{i} P\left(N_{j} \mid E_{i}\right) P_{0}\left(E_{i}\right)}$.

The prior $P_{0}\left(E_{i}\right)$ is the probability for a single event to fall into the $i$-th energy bin. Once the unfolding matrix is known, a first estimation of the spectrum can be produced:

$\hat{E}_{i}=\sum_{j} P\left(E_{i} \mid N_{j}\right) N_{j}$.

The normalized values of $\hat{E}_{i}$ are used iteratively as the new set of probabilities $P\left(E_{i}\right)$, in order to obtain an updated value of $P\left(E_{i} \mid N_{j}\right)$ and therefore of $\hat{E}_{i}$. The particular choice of the prior and the number of iterations may cause a small bias on the shape of the unfolded spectrum. A small number of iteration may not reflect the information given by the data, while a high number of iteration may amplify statistical fluctuations and distort the spectrum. Since the Bayesian method is strongly data driven, the effect of the particular choice of the prior is in general small, but is still taken into account as a source of systematic uncertainty. The prior should reflect, in principle, the best knowledge of the primary spectrum. The minimum bias is then achieved by adopting the true MC distribution. The strong data-driven nature of the iterative Bayesian method ensures very good results after few iterations. In this work, two iterations have been performed. A soft smoothing is applied to the first value of the probability $P_{1}\left(E_{i}\right)$. As prior distribution, the HKKM14 model has been used. Further details are given in Sect. 4.5.

Figure 6 shows the likelihood matrix for both $v_{e}$ and $v_{\mu}$ events, evaluated according to the binning described in Sect. 4.3 and including the contribution of the background.

\subsection{Uncertainties}

The total uncertainty on the atmospheric neutrino spectrum reconstruction is evaluated in each energy bin, including both contributions from statistics and systematic effects.

Statistics The statistical uncertainty is due to the stochastic fluctuations that occur in the data bins. The amount of this fluctuations is visible in Fig. 5, for each observable bin. In order to evaluate their impact in the final unfolded spectrum, 1000 toy data sets have been generated, each time varying the bin content according to a Poisson distribution. The final distribution in each bin of the unfolded spectrum is then fit with a Gaussian function, whose $\sigma$ is quoted as the statistical uncertainty. The statistical contribution ranges from $5 \%$ in
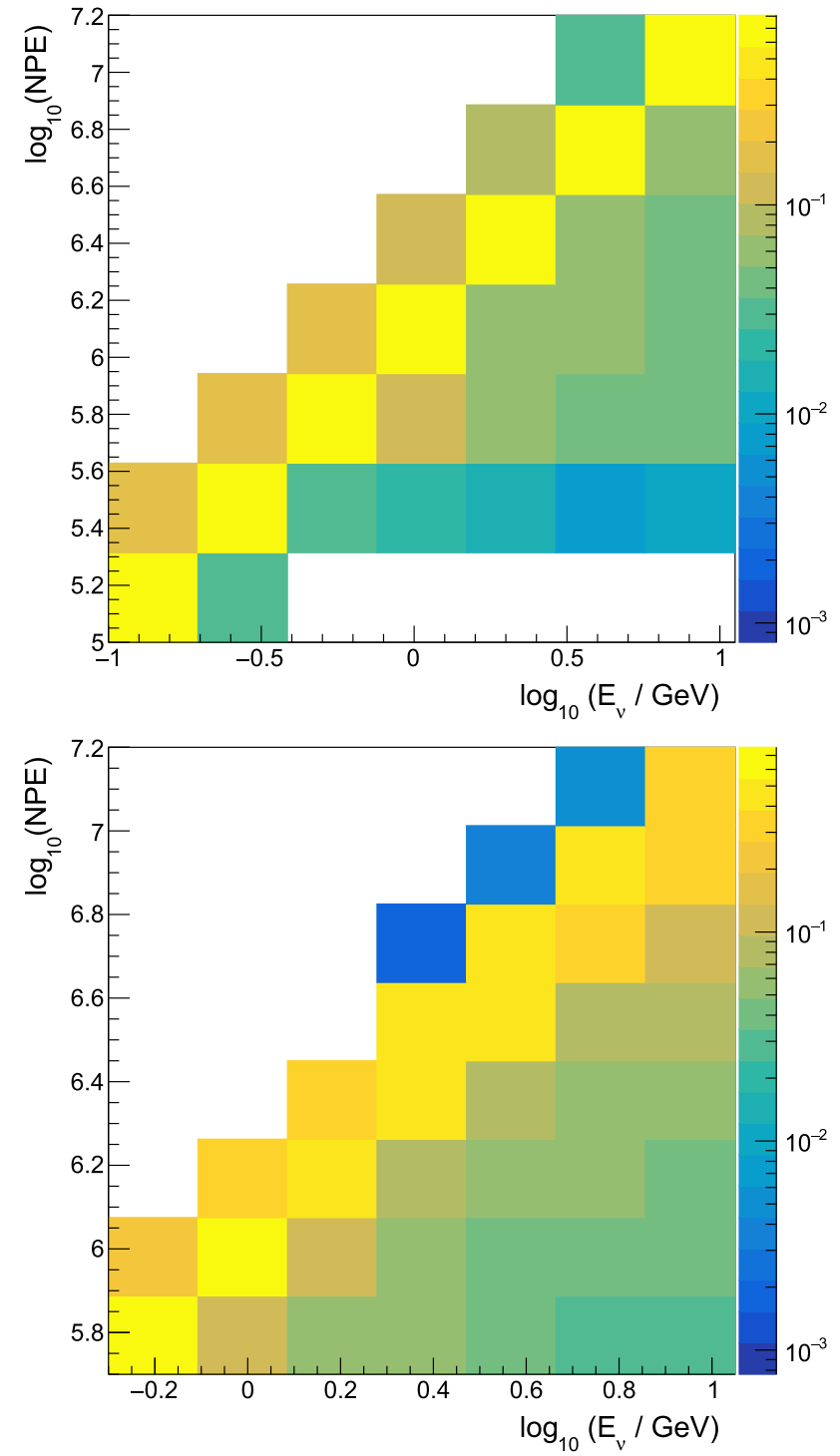

Fig. 6 Likelihood matrix for $v_{e}$ (top) and $v_{\mu}$ events (bottom)

the bins with highest statistics up to $\sim 15 \%$ in the highestenergy bins.

Selection criteria The selection procedure is in general not intended to produce any bias on the final sample. As explained in Sect. 4.1, fiducial cuts have been used in the unfolding procedure in order to improve the accuracy of the probability evaluations. The energy range of the final reconstructed spectrum is well contained inside the energy range of the Monte Carlo generated events, guaranteeing that the fiducial cuts do not introduce any bias. The neutrino flavor identification based on the time residual selection, on the other hand, could bring some uncertainty in the data bins where the statistics is low: an even small variation in the chosen cut value of $\sigma\left(t_{\text {res }}\right)$ could result in a substantially different value of the unfolded flux, due to the wide stochastic fluctuations. The whole analysis has been therefore per- 
Table 2 Best-fit neutrino oscillation parameters with associated $3 \sigma$ experimental uncertainty. Results are given both assuming normal ordering and inverted ordering. Table is taken from [39]

\begin{tabular}{lll}
\hline Parameter & $\mathrm{NH}$ & $\mathrm{IH}$ \\
\hline $\sin ^{2} \theta_{12}$ & $0.297_{-0.047}^{+0.057}$ & \\
$\sin ^{2} \theta_{23}$ & $0.425_{-0.044}^{+0.19}$ & $0.589_{-0.205}^{+0.047}$ \\
$\sin ^{2} \theta_{13}$ & $0.0215_{-0.0025}^{+0.0025}$ & $0.0216_{-0.0026}^{+0.0026}$ \\
$\delta / \pi$ & $1.38_{-0.38}^{+0.52}(2 \sigma)$ & $1.31_{-0.39}^{+0.57}(2 \sigma)$ \\
$\Delta m_{21}^{2} / 10^{-5} \mathrm{eV}^{2}$ & $7.37_{-0.44}^{+0.59}$ & \\
$\Delta m_{32}^{2} / 10^{-3} \mathrm{eV}^{2}$ & $2.56_{-0.12}^{+0.13}$ & $2.54_{-0.12}^{+0.12}$ \\
\hline
\end{tabular}

formed by varying the nominal cut value of $\sigma\left(t_{\text {res }}\right)$ by $1 \mathrm{~ns}$ steps in a $[-5 \mathrm{~ns},+5 \mathrm{~ns}]$ time window. The differences in the unfolded flux are relevant in the bins with less statistics, for the reasons explained above. The total contribution to the bin uncertainty is evaluated as the standard deviation of the flux values distribution in each bin.

Flavor oscillation The current uncertainties on the global fit oscillation parameters are reported in Table 2, which are assumed to be Gaussian. A toy MC has been used to generate 1000 data sets, randomly varying the oscillation parameters within the experimental uncertainties, including the mass ordering and assuming no correlation. The final distribution in the unfolded flux is fit in each bin with a Gaussian function. Since the resulting dispersion is rather small in every bin, the total per-bin uncertainty contribution is quoted as the displacement of the distribution fit peak with respect to the nominal flux value. The total contribution from oscillation parameters uncertainty is estimated to be below $1 \%$ on the entire spectrum. The only exception is the first bin of the $v_{\mu}$ spectrum, where oscillation effects are not negligible, which results into an uncertainty corresponding to a $\sigma$ of $1.2 \%$.

Cross-section The uncertainties on neutrino cross-section impact directly on the number of observed events. In the MC simulation process, as described in Sect. 3, neutrino interactions are managed by the GENIE software. The full list of uncertainty sources considered by GENIE is provided in [41]. A comprehensive handling of the whole list is not trivial, since it requires the simultaneous calculation of modified interaction probabilities in a wide parameter space. In this study, the evaluation of the cross-section uncertainty is based on experimental measurements provided by the T2K Collaboration [46-48], extrapolated from the associated data releases. Assuming the uncertainty on the measured cross section values to be Gaussian, the related visible spectrum is modified accordingly, within $1 \sigma$ interval. The propagated uncertainty on the unfolded flux is evaluated by unfolding 1000 toy MC data sets, with NPE bin contents altered according to random variations of the cross section parameters. The unfolded spectra distributions are then fit in each bin with a
Gaussian function, whose $\sigma$ is quoted as the related uncertainty contribution. The uncertainty in the neutrino crosssection values has a large impact in the final reconstructed flux, up to $20 \%$.

Unfolding procedure Although the iterative Bayesian unfolding method is data-driven, the particular MC sample may have an influence on the final result. This means that the initial estimation of the likelihood matrix may have an intrinsic bias, as well as the choice of the prior. The relative impact should be small, but it can have an impact in the unfolding bins with low statistics. The net effect cannot be exactly computed, but a reliable estimation can be achieved by unfolding modified data sets, generated by assuming a primary MC distribution reasonably far from the one used to evaluate the probabilities.

The modified spectra are produced from the original MC by means of a re-weighting procedure. The new spectrum can be expressed in the $i$-th unfolding bin as:

$\Phi_{v_{i}}^{\mathrm{MOD}}=(1+\alpha)\left(\frac{E_{v_{i}}}{1 \mathrm{GeV}}\right)^{\gamma} \Phi_{v_{i}}^{\mathrm{MC}}$,

where $\alpha$ acts on the absolute normalization and $\gamma$ on the shape of the primary spectrum. These two parameters are considered to range in the following intervals: \pm 0.05 for $\alpha$ and \pm 0.2 for $\gamma$.

The size of variation corresponds approximately to a $1 \sigma$ uncertainty interval in the predicted spectra. In Fig. 7 the comparison between each toy data sample and the corresponding unfolding result is reported, together with the fractional deviation between the input and the unfolded result. The deviation is below $1 \%$ and turns out to be slightly higher in the case of maximum variation of $\alpha$ and $\gamma$ in the bins with lower statistics. The conditional probabilities used in the unfolding procedure have been carefully evaluated by using different methods for both $v_{e}$ and $v_{\mu}$ samples. The relative deviations obtained for different energy bins and different NPE bins are reported in Table 3, as an example, for the $v_{e}$ sample. The effect on the obtained spectra turns out to be negligible.

The contributions of each uncertainty source are reported in Fig. 8, for each unfolding bin. The total uncertainty reported is calculated as the sum in quadrature of all contributions. The neutrino cross-section uncertainty represents the dominant contribution over the whole unfolded spectrum. The statistical uncertainty has also an important weight in high-energy bins. The total flux uncertainty ranges from a minimum value of $10-15 \%$ in the $\mathcal{O}(1 \mathrm{GeV})$ energy region, up to a $20-25 \%$ in the edge bins. 

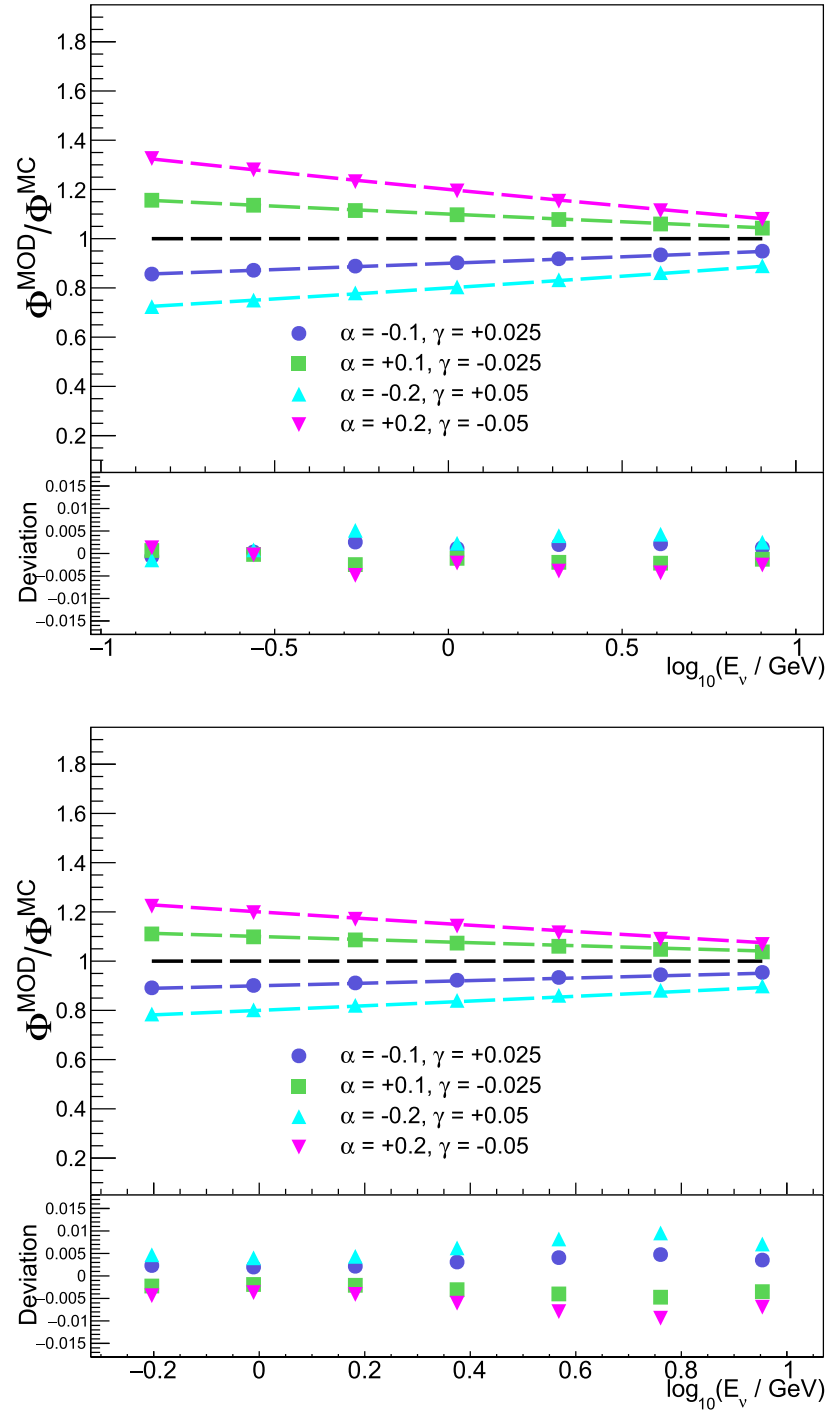

Fig. 7 Top panels: modified unfolded spectra (markers), together with the corresponding input (dashed lines). Bottom panels: relative deviation. Top: $v_{e}$ spectra; bottom: $v_{\mu}$ spectra. $\Phi^{M C}$ represents the nominal flux model [33], which is reported as the black dashed line. Four sets of modified spectra are plotted, whose $\alpha$ and $\gamma$ values are reported in the figures

Table 3 Relative deviations of the conditional probabilities evaluated by using the $v_{e}$ sample

\begin{tabular}{llll}
\hline $\log _{10}(\mathrm{NPE})$ & & & \\
$\log _{10}\left(E_{v} / \mathrm{GeV}\right)$ & -1 to -0.5 & -0.5 to 0.36 & 0.36 to 1.1 \\
\hline $5-5.5$ & 0.030 & 0.055 & 0.040 \\
$5.5-6.3$ & - & 0.005 & 0.075 \\
$6.3-7.2$ & - & - & 0.005 \\
\hline
\end{tabular}
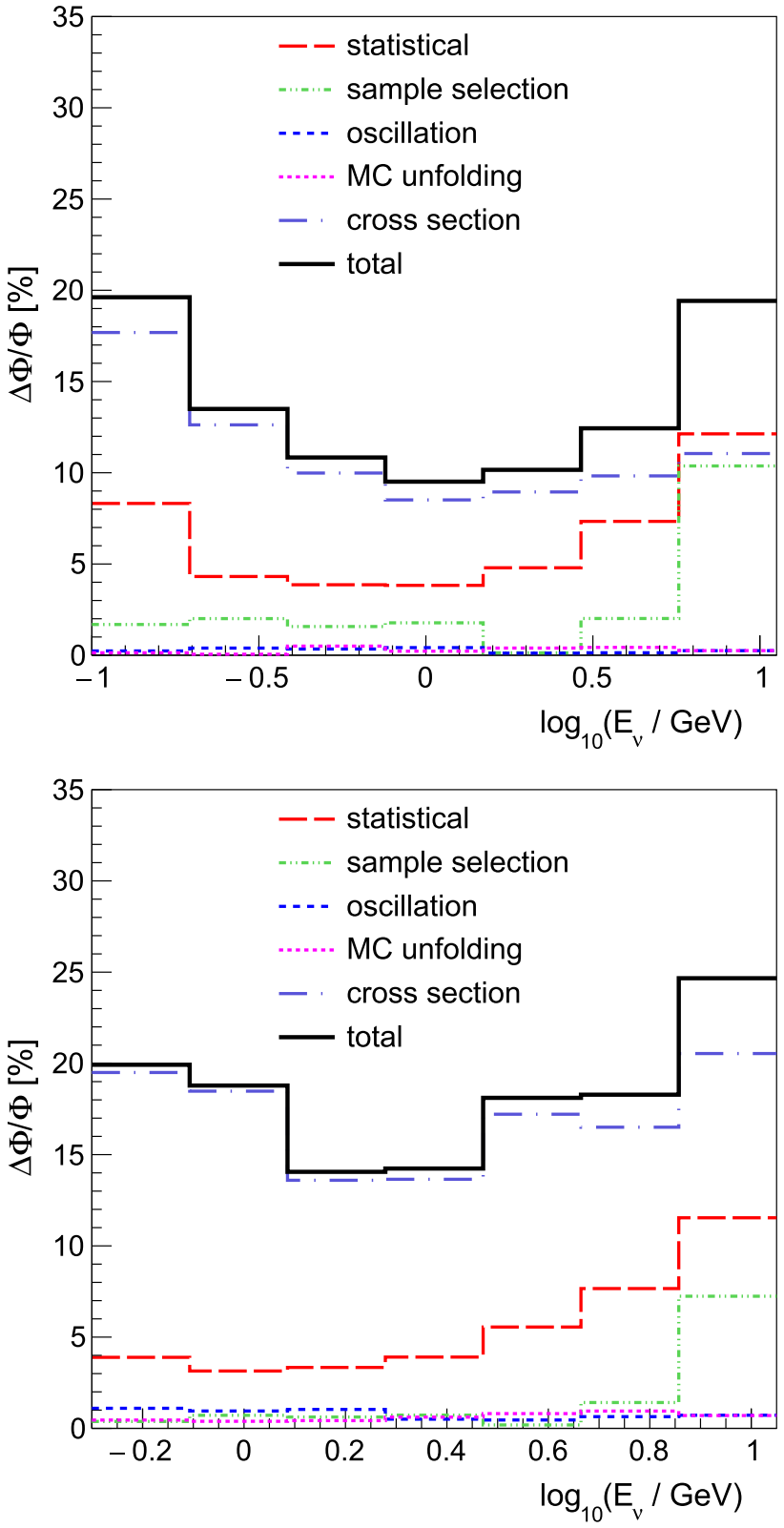

Fig. 8 Summary of estimated relative uncertainty on the unfolded flux, reported separately for each source. The total relative uncertainty is also reported. Top: $v_{e}$ spectrum; bottom: $v_{\mu}$ spectrum

\section{Results and discussion}

The unfolded $v_{e}$ and $v_{\mu}$ energy spectra are shown In Fig. 9. The binning is described in Sect. 4.4. The predicted HKKM14 flux [33] is also reported, both at the source and including oscillation effects along the baseline. The oscillation-induced flux deficit in the $v_{\mu}$ flux below $10 \mathrm{GeV}$ is clearly visible. 

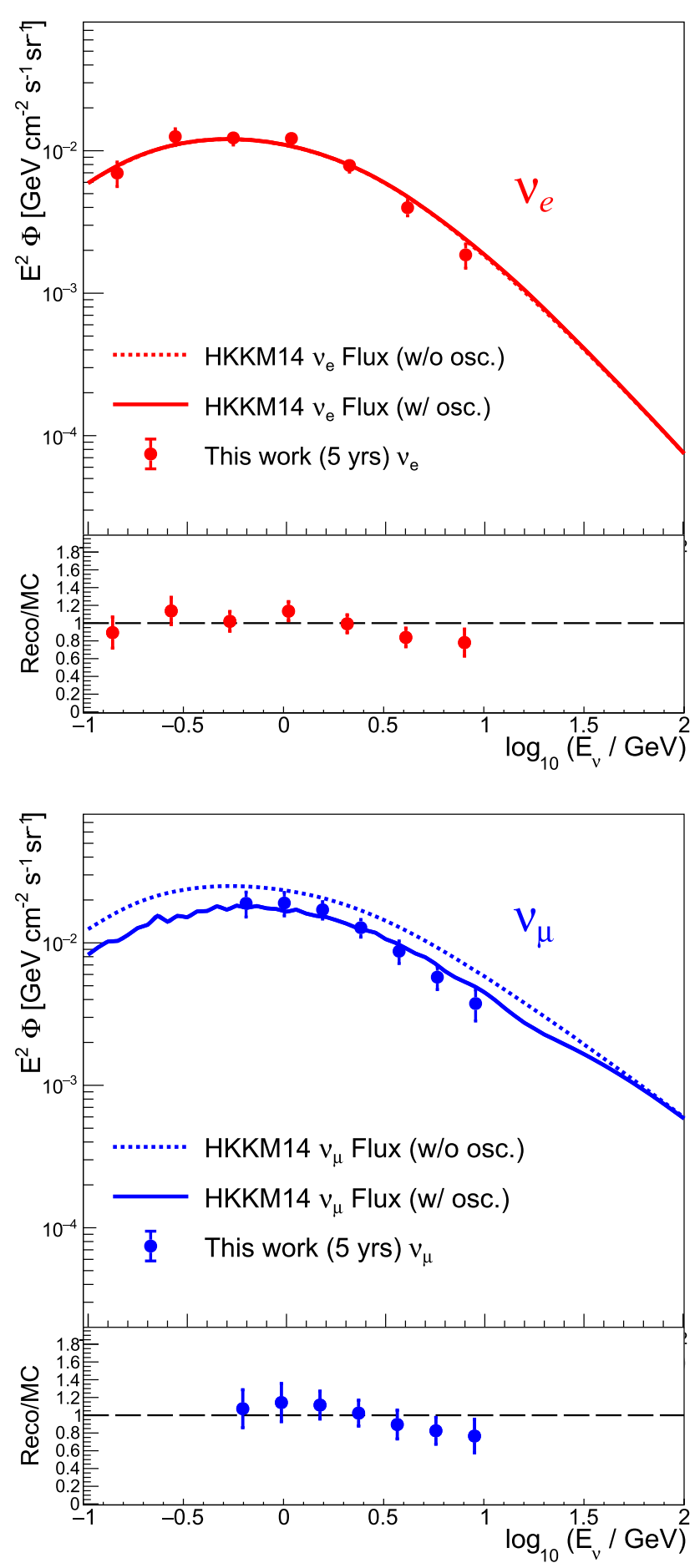

Fig. 9 Reconstructed energy spectra for the $v_{e}$ (top) and the $v_{\mu}$ flux (bottom). The fluxes are plotted multiplied by $E^{2}$, to give a better picture. The error bars on the flux values include all statistical and systematic contributions evaluated in Sect. 4.5. The HKKM14 flux prediction [33] is also reported, at the source (dashed line) and including the oscillation effects (full line)

JUNO is able to reconstruct the energy spectrum of atmospheric neutrinos in the energy range $[100 \mathrm{MeV}-10 \mathrm{GeV}]$, usually referred to as the "low-energy" region. This work, although based on simulated data only, shows the good capabilities of a large LS based detector like JUNO to measure the atmospheric neutrino flux. The energy region considered is already populated by other measurements, however some discrepancies still remain. JUNO can provide additional information about this interesting energy region, helping models in constraining their predictions. The quoted uncertainty is competitive with present experimental results and shows a margin of improvement by the increase of exposure time. Although JUNO's design is not optimized for atmospheric neutrino physics,

the extremely good performances in the atmospheric neutrino energy reconstruction can be fully exploited for the measurement of the energy spectrum. Moreover, atmospheric neutrinos are a natural source which will be fully accessible from the beginning of data taking.

\section{Conclusions}

The JUNO detector has been designed from the beginning as a state-of-the-art detector for neutrino physics. The large dimensions of the detector, as well as its dense instrumentation, pave the way to an entire series of measurements, in a multi-purpose approach. The atmospheric neutrino flux is a natural source that can be observed, from the very beginning of data-taking. Although the detector design is not optimized for this class of events, the large active volume and the fine energy resolution allow to reconstruct the energy spectrum with a competitive precision, especially in the low-energy region.

In this work, a large set of MC events has been generated to evaluate the detector performances. A smaller set has been used to simulate the real data. Thanks to the timing performances of JUNO, the flavor of primary neutrinos can be separated with a limited residual contamination. A rejection power of the order of $10^{5}$ has been applied to reduce atmospheric muon background. The atmospheric neutrino energy spectrum has been reconstructed in the energy range $[100 \mathrm{MeV}-10 \mathrm{GeV}]$, separately for $v_{e}$ and $v_{\mu}$, assuming a $\sim 5$ years detector livetime. The reconstructed spectra lie inside an interesting energy region, where previous measurements show some discrepancies. The results obtained show the good performance of JUNO in detecting the atmospheric neutrino flux in the low energy region, where theoretical models have large uncertainties. The inferred information can provide a fruitful input to constrain flux predictions, which are essential to evaluate the impact of atmospheric neutrinos in the search of rare events.

Acknowledgements We are grateful for the ongoing cooperation from the China General Nuclear Power Group. This work was supported by the Chinese Academy of Sciences, the National Key R\&D Program of 
China, the CAS Center for Excellence in Particle Physics, Wuyi University, and the Tsung-Dao Lee Institute of Shanghai Jiao Tong University in China, the Institut National de Physique Nucléaire et de Physique de Particules (IN2P3) in France, the Istituto Nazionale di Fisica Nucleare (INFN) in Italy, the Italian-Chinese collaborative research program MAECI-NSFC, the Fond de la Recherche Scientifique (F.R.S-FNRS) and FWO under the "Excellence of Science - EOS in Belgium, the Conselho Nacional de Desenvolvimento Científico e Tecnològico in Brazil, the Agencia Nacional de Investigacion y Desarrollo in Chile, the Charles University Research Centre and the Ministry of Education, Youth, and Sports in Czech Republic, the Deutsche Forschungsgemeinschaft (DFG), the Helmholtz Association, and the Cluster of Excellence PRISMA+ in Germany, the Joint Institute of Nuclear Research (JINR) and Lomonosov Moscow State University in Russia, the joint Russian Science Foundation (RSF) and National Natural Science Foundation of China (NSFC) research program, the MOST and MOE in Taiwan, the Chulalongkorn University and Suranaree University of Technology in Thailand, and the University of California at Irvine in USA.

Data Availability Statement This manuscript has no associated data or the data will not be deposited. [Authors' comment: This work was based on Monte Carlo studies with the JUNO software, thus no data will be deposited.]

Open Access This article is licensed under a Creative Commons Attribution 4.0 International License, which permits use, sharing, adaptation, distribution and reproduction in any medium or format, as long as you give appropriate credit to the original author(s) and the source, provide a link to the Creative Commons licence, and indicate if changes were made. The images or other third party material in this article are included in the article's Creative Commons licence, unless indicated otherwise in a credit line to the material. If material is not included in the article's Creative Commons licence and your intended use is not permitted by statutory regulation or exceeds the permitted use, you will need to obtain permission directly from the copyright holder. To view a copy of this licence, visit http://creativecomm ons.org/licenses/by/4.0/.

Funded by SCOAP ${ }^{3}$.

\section{References}

1. G.D. Barr, T.K. Gaisser, P. Lipari, S. Robbins, T. Stanev, Threedimensional calculation of atmospheric neutrinos. Phys. Rev. D 70, 023006 (2004). https://doi.org/10.1103/PhysRevD.70.023006

2. M. Honda, T. Kajita, K. Kasahara, S. Midorikawa, T. Sanuki, Calculation of atmospheric neutrino flux using the interaction model calibrated with atmospheric muon data. Phys. Rev. D 75, 043006 (2007). https://doi.org/10.1103/PhysRevD.75.043006

3. T.K. Gaisser, M. Honda, Flux of Atmospheric Neutrinos. Annu. Rev. Nucl. Part. Sci. 52(1), 153-199 (2002). https://doi.org/10. 1146/annurev.nucl.52.050102.090645

4. M. Guan, et al., A parametrization of the cosmic-ray muon flux at sea-level (2015). arXiv:1509.06176

5. T.K. Gaisser, Cosmic Rays and Particle Physics (1991)

6. J.R. Hoerandel, Models of the knee in the energy spectrum of cosmic rays. Astropart. Phys. 21, 241-265 (2004). https://doi.org/10. 1016/j.astropartphys.2004.01.004. arXiv:astro-ph/0402356

7. E. Richard et al., Measurements of the atmospheric neutrino flux by Super-Kamiokande: Energy spectra, geomagnetic effects, and solar modulation. Phys. Rev. D 94, 052001 (2016). https://doi.org/ 10.1103/PhysRevD.94.052001

8. M.G. Aartsen et al., Measurement of the atmospheric $v_{e}$ flux in IceCube. Phys. Rev. Lett. 110, 151105 (2013). https://doi.org/10. 1103/PhysRevLett.110.151105
9. M.G. Aartsen et al., Measurement of the atmospheric $v_{e}$ Spectrum with IceCube. Phys. Rev. D 91, 122004 (2015). https://doi.org/10. 1103/PhysRevD.91.122004

10. R. Abbasi et al., Measurement of the atmospheric neutrino energy spectrum from $100 \mathrm{GeV}$ to $400 \mathrm{TeV}$ with IceCube. Phys. Rev. D 83, 012001 (2011). https://doi.org/10.1103/PhysRevD.83.012001

11. K. Daum et al., Determination of the atmospheric neutrino spectra with the Fréjus detector. Z. Für Phys. C Part. Fields 66(3), 417-428 (1995). https://doi.org/10.1007/BF01556368

12. R. Enberg, M.H. Reno, I. Sarcevic, Prompt neutrino fluxes from atmospheric charm. Phys. Rev. D 78, 043005 (2008). https://doi. org/10.1103/PhysRevD.78.043005

13. M.R.A.D. Martin, A. Stasto, Prompt neutrinos from atmospheric c-cbar and b-bbar production and the gluon at very small x. Acta Phys. Polon. B 34, 3273 (2003)

14. G. Fiorentini, V.A. Naumov, F.L. Villante, Atmospheric neutrino flux supported by recent muon experiments. Phys. Lett. B 510(1), 173-188 (2001). https://doi.org/10.1016/ S0370-2693(01)00572-X

15. M. Aartsen et al., Measurement of atmospheric neutrino oscillations at $6-56 \mathrm{GeV}$ with IceCube DeepCore. Phys. Rev. Lett. 120, 071801 (2018). https://doi.org/10.1103/PhysRevLett.120.071801

16. C. Achar et al., Detection of muons produced by cosmic ray neutrinos deep underground. Phys. Lett. 18(2), 196-199 (1965). https:// doi.org/10.1016/0031-9163(65)90712-2

17. F. Reines et al., Evidence for High-Energy Cosmic-Ray Neutrino Interactions. Phys. Rev. Lett. 15, 429-433 (1965). https://doi.org/ 10.1103/PhysRevLett.15.429

18. Y. Fukuda et al., Evidence for oscillation of atmospheric neutrinos. Phys. Rev. Lett. 81, 1562-1567 (1998). https://doi.org/10.1103/ PhysRevLett.81.1562

19. B. Abi, et al., Deep Underground Neutrino Experiment (DUNE), Far Detector Technical Design Report, volume II: DUNE Physics (2020). arXiv:2002.03005

20. H.-K. Proto-Collaboration, K. Abe et al., Hyper-Kamiokande Design Report (2018). arXiv:1805.04163

21. A. Kumar et al., Physics potential of the ICAL detector at the Indiabased Neutrino Observatory (INO), Pramana. J. Phys. 88(5), 79 (2017). https://doi.org/10.1007/s12043-017-1373-4

22. S. Adrián-Martínez et al., Letter of intent for KM3net 2.0. J. Phys. G Nucl. Part. Phys. 43(8), 084001 (2016). https://doi.org/10.1088/ 0954-3899/43/8/084001

23. M. G. Aartsen et al., PINGU: a vision for neutrino and particle physics at the South Pole. J. Phys. G Nucl. Part. Phys. 44(5), 054006 (2017). https://doi.org/10.1088/1361-6471/44/5/054006

24. M.G. Aartsen et al., Measurement of the $v_{\mu}$ energy spectrum with IceCube-79. Eur. Phys. J. C 77(10), 692 (2017). https://doi.org/10. 1140/epjc/s10052-017-5261-3

25. F. An et al., Neutrino physics with JUNO. J. Phys. G Nucl. Part. Phys. 43(3), 030401 (2016). https://doi.org/10.1088/0954-3899/ 43/3/030401

26. T. Adam, et al., JUNO Conceptual Design Report (2015). arXiv: 1508.07166

27. C. Cao et al., Mass production and characterization of 3-inch PMTs for the JUNO experiment. Nucl. Instrum. Methods A (2021). arXiv:2102.11538

28. T. Adam et al., The OPERA experiment target tracker. Nucl. Instrum. Methods Phys. Res. Sect. A 577(3), 523-539 (2007). https://doi.org/10.1016/j.nima.2007.04.147

29. A. Abusleme, et al., Optimization of the JUNO liquid scintillator composition using a Daya Bay antineutrino detector. Nucl. Instrum. Methods Phys. Res. Sect. A Accel. Spectrom. Detect. Assoc. Equip., 164823 (2020). https://doi.org/10.1016/j. nima.2020.164823 
30. P. Lombardi et al., Distillation and stripping pilot plants for the JUNO neutrino detector: design, operations and reliability. Nucl. Instrum. Methods Phys. Res. Sect. A 925, 6-17 (2019). https://doi. org/10.1016/j.nima.2019.01.071

31. A. Abusleme et al., Calibration strategy of the JUNO Experiment. JHEP (2020). arXiv:2011.06405

32. J.A. Formaggio, G.P. Zeller, From ev to EefV: Neutrino cross sections across energy scales. Rev. Mod. Phys. 84, 1307-1341 (2012). https://doi.org/10.1103/RevModPhys.84.1307

33. M. Honda, M.S. Athar, T. Kajita, K. Kasahara, S. Midorikawa, Atmospheric neutrino flux calculation using the NRLMSISE-00 atmospheric model. Phys. Rev. D 92, 023004 (2015). https://doi. org/10.1103/PhysRevD.92.023004

34. T. Sanuki et al., Precise measurement of cosmic-ray proton and helium spectra with the BESS spectrometer. Astrophys. J. 545(2), 1135-1142 (2000). https://doi.org/10.1086/317873

35. S. Haino et al., Measurements of primary and atmospheric cosmicray spectra with the BESS-TeV spectrometer. Phys. Lett. B 594(1), 35-46 (2004). https://doi.org/10.1016/j.physletb.2004.05.019

36. J. Alcaraz et al., Protons in near Earth orbit. Phys. Lett. B 472(1), 215-226 (2000). https://doi.org/10.1016/S0370-2693(99)01427-6

37. S. Roesler, R. Engel, J. Ranft, The Monte Carlo Event Generator DPMJET-III, Advanced Monte Carlo for Radiation Physics. Part. Transp. Simul. Appl., 1033-1038 (2001). https://doi.org/10.1007/ 978-3-642-18211-2_166

38. K. Niita, T. Sato, H. Iwase, H. Nose, H. Nakashima, L. Sihver, Phits-a particle and heavy ion transport code system. Radiat. Meas. 41(9), 1080-1090 (2006). https://doi.org/10.1016/j.radmeas.2006. 07.013 (space Radiation Transport, Shielding, and Risk Assessment Models)

39. M. Tanabashi et al., Review of particle physics. Phys. Rev. D 98, 030001 (2018). https://doi.org/10.1103/PhysRevD.98.030001
40. C. Andreopoulos et al., The GENIE neutrino Monte Carlo generator. Nucl. Instrum. Methods Phys. Res. Sect. A 614(1), 87-104 (2010). https://doi.org/10.1016/j.nima.2009.12.009

41. C. Andreopoulos, et al., The GENIE Neutrino Monte Carlo Generator: physics and user manual (2015). arXiv: 1510.05494

42. J.H. Zou et al., SNiPER: an offline software framework for noncollider physics experiments. J. Phys. Conf. Ser. 664(7), 072053 (2015). https://doi.org/10.1088/1742-6596/664/7/072053

43. V. Kudryavtsev, Muon simulation codes MUSIC and MUSUN for underground physics. Comput. Phys. Commun. 180(3), 339-346 (2009). https://doi.org/10.1016/j.cpc.2008.10.013

44. G. Settanta et al., e- $\mu$ discrimination at high energy in the JUNO detector. EPJ Web Conf. 209, 01011 (2019). https://doi.org/10. 1051/epjconf/201920901011

45. G. DAgostini, A multidimensional unfolding method based on Bayes theorem. Nucl. Instrum. Methods Phys. Res. Sect. A Accel. Spectrom. Detect. Assoc. Equip. 362(2), 487-498 (1995). https:// doi.org/10.1016/0168-9002(95)00274-X

46. K. Abe et al., Measurement of the inclusive electron neutrino charged current cross section on carbon with the T2K near detector. Phys. Rev. Lett. 113, 241803 (2014). https://doi.org/10.1103/ PhysRevLett.113.241803

47. K. Abe et al., Measurement of the $v_{\mu}$ charged-current quasielastic cross section on carbon with the ND280 detector at T2K. Phys. Rev. D 92, 112003 (2015). https://doi.org/10.1103/PhysRevD.92. 112003

48. K. Abe et al., First measurement of the muon neutrino charged current single pion production cross section on water with the T2K near detector. Phys. Rev. D 95, 012010 (2017). https://doi.org/10. 1103/PhysRevD.95.012010

\section{The JUNO Collaboration}

Angel Abusleme ${ }^{5}$, Thomas Adam ${ }^{45}$, Shakeel Ahmad ${ }^{66}$, Rizwan Ahmed ${ }^{66}$, Sebastiano Aiello ${ }^{55}$, Muhammad Akram ${ }^{66}$, Fengpeng $\mathrm{An}^{29}$, Guangpeng $\mathrm{An}^{10}$, Qi $\mathrm{An}^{22}$, Giuseppe Andronico ${ }^{55}$, Nikolay Anfimov ${ }^{67}$, Vito Antonelli ${ }^{57}$, Tatiana Antoshkina ${ }^{67}$, Burin Asavapibhop ${ }^{71}$, João Pedro Athayde Marcondes de André ${ }^{45}$, Didier Auguste ${ }^{43}$, Andrej Babic ${ }^{70}$, Wander Baldini ${ }^{56}$, Andrea Barresi ${ }^{58}$, Eric Baussan ${ }^{45}$, Marco Bellato ${ }^{60}$, Antonio Bergnoli ${ }^{60}$, Enrico Bernieri ${ }^{64}$, Thilo Birkenfeld ${ }^{48}$, Sylvie Blin $^{43}$, David Blum ${ }^{54}$, Simon Blyth ${ }^{40}$, Anastasia Bolshakova ${ }^{67}$, Mathieu Bongrand ${ }^{47}$, Clément Bordereau ${ }^{44,40}$, Dominique Breton $^{43}$, Augusto Brigatti ${ }^{57}$, Riccardo Brugnera ${ }^{61}$, Riccardo Bruno ${ }^{55}$, Antonio Budano ${ }^{64}$, Mario Buscemi ${ }^{55}$, Jose Busto ${ }^{46}$, Ilya Butorov ${ }^{67}$, Anatael Cabrera ${ }^{43}, \mathrm{Hao} \mathrm{Cai}^{34}, \mathrm{Xiao} \mathrm{Cai}^{10}$, Yanke Cai $^{10}$, Zhiyan Cai ${ }^{10}$, Antonio Cammi ${ }^{59}$, Agustin Campeny ${ }^{5}$, Chuanya Cao ${ }^{10}$, Guofu Cao ${ }^{10}$, Jun Cao ${ }^{10}$, Rossella Caruso ${ }^{55}$, Cédric Cerna ${ }^{44}$, Jinfan Chang ${ }^{10}$, Yun Chang ${ }^{39}$, Pingping Chen ${ }^{18}$, Po-An Chen ${ }^{40}$, Shaomin Chen ${ }^{13}$, Xurong Chen ${ }^{26}$, Yi-Wen $\mathrm{Chen}^{38}$, Yixue Chen ${ }^{11}$, Yu Chen ${ }^{20}$, Zhang Chen ${ }^{10}$, Jie Cheng ${ }^{10}$, Yaping Cheng ${ }^{7}$, Alexey Chetverikov ${ }^{67}$, Davide Chiesa ${ }^{58}$, Pietro Chimenti ${ }^{3}$, Artem Chukanov ${ }^{67}$, Gérard Claverie ${ }^{44}$, Catia Clementi $^{62}$, Barbara Clerbaux ${ }^{2}$, Selma Conforti Di Lorenzo ${ }^{43}$, Daniele Corti ${ }^{60}$, Salvatore Costa ${ }^{55}$, Flavio Dal Corso ${ }^{60}$, Olivia Dalager $^{74}$, Christophe De La Taille ${ }^{43}$, Jiawei Deng ${ }^{34}$, Zhi Deng ${ }^{13}$, Ziyan Deng ${ }^{10}$, Wilfried Depnering ${ }^{52}$, Marco Diaz ${ }^{5}$, Xuefeng Ding ${ }^{57}$, Yayun Ding ${ }^{10}$, Bayu Dirgantara ${ }^{73}$, Sergey Dmitrievsky ${ }^{67}$, Tadeas Dohnal ${ }^{41}$, Dmitry Dolzhikov ${ }^{67}$, Georgy Donchenko $^{69}$, Jianmeng Dong ${ }^{13}$, Evgeny Doroshkevich ${ }^{68}$, Marcos Dracos ${ }^{45}$, Frédéric Druillole ${ }^{44}$, Shuxian Du ${ }^{37}$, Stefano Dusini $^{60}$, Martin Dvorak ${ }^{41}$, Timo Enqvist ${ }^{42}$, Heike Enzmann ${ }^{52}$, Andrea Fabbri ${ }^{64}$, Lukas Fajt ${ }^{70}$, Donghua Fan ${ }^{24}$, Lei Fan ${ }^{10}$, Can Fang ${ }^{28}$, Jian Fang ${ }^{10}$, Wenxing Fang ${ }^{10}$, Marco Fargetta ${ }^{55}$, Dmitry Fedoseev ${ }^{67}$, Vladko Fekete ${ }^{70}$, Li-Cheng Feng ${ }^{38}$, Qichun Feng $^{21}$, Richard Ford ${ }^{57}$, Andrey Formozov ${ }^{57}$, Amélie Fournier ${ }^{44}$, Haonan $\mathrm{Gan}^{32}$, Feng Gao ${ }^{48}$, Alberto Garfagnini ${ }^{61}$, Christoph Genster $^{50}$, Marco Giammarchi ${ }^{57}$, Agnese Giaz ${ }^{61}$, Nunzio Giudice ${ }^{55}$, Maxim Gonchar ${ }^{67}$, Guanghua Gong ${ }^{13}$, Hui Gong ${ }^{13}$, Oleg Gorchakov $^{67}$, Yuri Gornushkin ${ }^{67}$, Alexandre Göttel ${ }^{50,48}$, Marco Grassi ${ }^{61}$, Christian Grewing ${ }^{51}$, Vasily Gromov ${ }^{67}$, Minghao $\mathrm{Gu}^{10}$, Xiaofei $\mathrm{Gu}^{37}$, Yu Gu${ }^{19}$, Mengyun Guan ${ }^{10}$, Nunzio Guardone ${ }^{55}$, Maria Gul ${ }^{66}$, Cong Guo ${ }^{10}$, Jingyuan Guo ${ }^{20}$, Wanlei Guo $^{10}$, Xinheng Guo ${ }^{8}$, Yuhang Guo ${ }^{35,50}$, Paul Hackspacher ${ }^{52}$, Caren Hagner ${ }^{49}$, Ran Han ${ }^{7}$, Yang Han ${ }^{43}$, Muhammad Sohaib Hassan $^{66}$, Miao He ${ }^{10}$, Wei He ${ }^{10}$, Tobias Heinz ${ }^{54}$, Patrick Hellmuth ${ }^{44}$, Yuekun Heng ${ }^{10}$, Rafael Herrera ${ }^{5}$, Daojin Hong ${ }^{28}$, YuenKeung $\mathrm{Hor}^{20}$, Shaojing Hou ${ }^{10}$, Yee Hsiung ${ }^{40}$, Bei-Zhen $\mathrm{Hu}^{40}$, Hang $\mathrm{Hu}^{20}$, Jianrun $\mathrm{Hu}^{10}$, Jun $\mathrm{Hu}^{10}$, Shouyang $\mathrm{Hu}^{9}$, 
Tao $\mathrm{Hu}^{10}$, Zhuojun $\mathrm{Hu}^{20}$, Chunhao Huang ${ }^{20}$, Guihong Huang ${ }^{10}$, Hanxiong Huang ${ }^{9}$, Qinhua Huang ${ }^{45}$, Wenhao Huang ${ }^{25}$, Xin Huang ${ }^{10}$, Xingtao Huang ${ }^{25}$, Yongbo Huang ${ }^{28}$, Jiaqi Hui ${ }^{30}$, Lei Huo ${ }^{21}$, Wenju Huo ${ }^{22}$, Cédric Huss ${ }^{44}$, Safeer Hussain ${ }^{66}$, Antonio Insolia ${ }^{55}$, Ara Ioannisian ${ }^{1}$, Roberto Isocrate ${ }^{60}$, Beatrice Jelmini ${ }^{61}$, Kuo-Lun Jen ${ }^{38}$, Ignacio Jeria ${ }^{5}$, Xiaolu Ji ${ }^{10}$, Xingzhao $\mathrm{Ji}^{20}$, Huihui $\mathrm{Jia}^{33}$, Junji Jia ${ }^{34}$, Siyu Jian ${ }^{9}$, Di Jiang ${ }^{22}$, Xiaoshan Jiang ${ }^{10}$, Ruyi $\mathrm{Jin}^{10}$, Xiaoping Jing ${ }^{10}$, Cécile Jollet ${ }^{44}$, Sirichok Jungthawan ${ }^{73}$, Leonidas Kalousis ${ }^{45}$, Philipp Kampmann ${ }^{50}$, Li Kang ${ }^{18}$, Michael Karagounis ${ }^{51}$, Narine Kazarian ${ }^{1}$, Waseem Khan ${ }^{35}$, Khanchai Khosonthongkee ${ }^{73}$, Denis Korablev ${ }^{67}$, Konstantin Kouzakov ${ }^{69}$, Alexey Krasnoperov ${ }^{67}$, Zinovy Krumshteyn ${ }^{67}$, Andre Kruth ${ }^{51}$, Nikolay Kutovskiy ${ }^{67}$, Pasi Kuusiniemi ${ }^{42}$, Tobias Lachenmaier ${ }^{54}$, Cecilia Landini ${ }^{57}$, Sébastien Leblanc $^{44}$, Victor Lebrin ${ }^{47}$, Frederic Lefevre ${ }^{47}$, Ruiting Lei ${ }^{18}$, Rupert Leitner ${ }^{41}$, Jason Leung ${ }^{38}$, Demin Li ${ }^{37}$, Fei Li $^{10}$, Fule $\mathrm{Li}^{13}$, Haitao $\mathrm{Li}^{20}$, Huiling $\mathrm{Li}^{10}$, Jiaqi $\mathrm{Li}^{20}$, Mengzhao $\mathrm{Li}^{10}$, Min $\mathrm{Li}^{11}$, Nan $\mathrm{Li}^{10}$, Nan $\mathrm{Li}^{16}$, Qingjiang $\mathrm{Li}^{16}, \mathrm{Ruhui} \mathrm{Li}^{10}$,

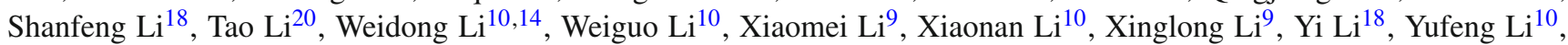
Zhaohan $\mathrm{Li}^{10}$, Zhibing $\mathrm{Li}^{20}$, Ziyuan $\mathrm{Li}^{20}$, Hao Liang ${ }^{9}$, Hao Liang ${ }^{22}$, Jingjing Liang ${ }^{28}$, Daniel Liebau ${ }^{51}$, Ayut Limphirat ${ }^{73}$, Sukit Limpijumnong ${ }^{73}$, Guey-Lin $\operatorname{Lin}^{38}$, Shengxin Lin ${ }^{18}$, Tao $\operatorname{Lin}^{10}$, Jiajie Ling ${ }^{20}$, Ivano Lippi ${ }^{60}$, Fang Liu ${ }^{11}$, Haidong Liu ${ }^{37}$, Hongbang $\mathrm{Liu}^{28}$, Hongjuan Liu ${ }^{23}$, Hongtao Liu ${ }^{20}$, Hui Liu ${ }^{19}$, Jianglai Liu ${ }^{30,31}$, Jinchang Liu ${ }^{10}$, Min Liu ${ }^{23}$, Qian Liu ${ }^{14}$, Qin $\mathrm{Liu}^{22}$, Runxuan Liu ${ }^{50,48}$, Shuangyu Liu ${ }^{10}$, Shubin Liu ${ }^{22}$, Shulin Liu ${ }^{10}$, Xiaowei Liu ${ }^{20}$, Xiwen Liu ${ }^{28}$, Yan Liu ${ }^{10}$, Yunzhe Liu ${ }^{10}$, Alexey Lokhov ${ }^{69,68}$, Paolo Lombardi ${ }^{57}$, Claudio Lombardo ${ }^{55}$, Kai Loo ${ }^{52}$, Chuan Lu $^{32}$, Haoqi Lu ${ }^{10}$, Jingbin Lu ${ }^{15}$, Junguang $\mathrm{Lu}^{10}$, Shuxiang $\mathrm{Lu}^{37}$, Xiaoxu Lu ${ }^{10}$, Bayarto Lubsandorzhiev ${ }^{68}$, Sultim Lubsandorzhiev ${ }^{68}$, Livia Ludhova ${ }^{50,48}$, Fengjiao Luo $^{10}$, Guang Luo ${ }^{20}$, Pengwei Luo ${ }^{20}$, Shu Luo ${ }^{36}$, Wuming Luo ${ }^{10}$, Vladimir Lyashuk ${ }^{68}$, Bangzheng Ma ${ }^{25}$, Qiumei Ma ${ }^{10}$, Si $\mathrm{Ma}^{10}$, Xiaoyan $\mathrm{Ma}^{10}$, Xubo Ma ${ }^{11}$, Jihane Maalmi ${ }^{43}$, Yury Malyshkin ${ }^{67}$, Fabio Mantovani ${ }^{56}$, Francesco Manzali ${ }^{61}$, Xin Mao ${ }^{7}$, Yajun Mao ${ }^{12}$, Stefano M. Mari ${ }^{64}$, Filippo Marini ${ }^{61}$, Sadia Marium ${ }^{66}$, Cristina Martellini ${ }^{64}$, Gisele Martin-Chassard ${ }^{43}$, Agnese Martini $^{63}$, Davit Mayilyan ${ }^{1}$, Ints Mednieks ${ }^{65}$, Yue Meng ${ }^{30}$, Anselmo Meregaglia ${ }^{44}$, Emanuela Meroni ${ }^{57}$, David Meyhöfer ${ }^{49}$, Mauro Mezzetto $^{60}$, Jonathan Miller ${ }^{6}$, Lino Miramonti ${ }^{57}$, Salvatore Monforte ${ }^{55}$, Paolo Montini ${ }^{64}$, Michele Montuschi ${ }^{56}$, Axel Müller ${ }^{54}$, Pavithra Muralidharan ${ }^{51}$, Massimiliano Nastasi ${ }^{58}$, Dmitry V. Naumov ${ }^{67}$, Elena Naumova ${ }^{67}$, Diana Navas-Nicolas ${ }^{43}$, Igor Nemchenok ${ }^{67}$, Minh Thuan Nguyen Thi ${ }^{38}$, Feipeng Ning ${ }^{10}$, Zhe Ning ${ }^{10}$, Hiroshi Nunokawa ${ }^{4}$, Lothar Oberauer ${ }^{53}$, Juan Pedro Ochoa-Ricoux ${ }^{74,5}$, Alexander Olshevskiy ${ }^{67}$, Domizia Orestano ${ }^{64}$, Fausto Ortica ${ }^{62}$, Rainer Othegraven ${ }^{52}$, Hsiao-Ru $\mathrm{Pan}^{40}$, Alessandro Paoloni ${ }^{63}$, Nina Parkalian ${ }^{51}$, Sergio Parmeggiano ${ }^{57}$, Yatian Pei ${ }^{10}$, Nicomede Pelliccia ${ }^{62}$, Anguo Peng ${ }^{23}$, Haiping Peng ${ }^{22}$, Frédéric Perrot ${ }^{44}$, Pierre-Alexandre Petitjean ${ }^{2}$, Fabrizio Petrucci ${ }^{64}$, Oliver Pilarczyk ${ }^{52}$, Luis Felipe Piñeres Rico $^{45}$, Artyom Popov ${ }^{69}$, Pascal Poussot ${ }^{45}$, Wathan Pratumwan ${ }^{73}$, Ezio Previtali ${ }^{58}$, Fazhi Qi ${ }^{10}$, Ming Qi ${ }^{27}$, Sen $_{\text {Qian }}{ }^{10}$, Xiaohui Qian ${ }^{10}$, Zhen Qian ${ }^{20}$, Hao Qiao ${ }^{12}$, Zhonghua Qin ${ }^{10}$, Shoukang Qiu ${ }^{23}$, Muhammad Usman Rajput ${ }^{66}$, Gioacchino Ranucci ${ }^{57}$, Neill Raper ${ }^{20}$, Alessandra Re ${ }^{57}$, Henning Rebber ${ }^{49}$, Abdel Rebii ${ }^{44}$, Bin Ren ${ }^{18}$, Jie Ren ${ }^{9}$, Taras Rezinko ${ }^{67}$, Barbara Ricci $^{56}$, Markus Robens ${ }^{51}$, Mathieu Roche ${ }^{44}$, Narongkiat Rodphai ${ }^{71}$, Aldo Romani ${ }^{62}$, Bedřich Roskovec ${ }^{74}$, Christian Roth ${ }^{51}$, Xiangdong Ruan ${ }^{28}$, Xichao Ruan ${ }^{9}$, Saroj Rujirawat ${ }^{73}$, Arseniy Rybnikov ${ }^{67}$, Andrey Sadovsky ${ }^{67}$, Paolo Saggese ${ }^{57}$, Giuseppe Salamanna ${ }^{64}$, Simone Sanfilippo ${ }^{64}$, Anut Sangka ${ }^{72}$, Nuanwan Sanguansak ${ }^{73}$, Utane Sawangwit ${ }^{72}$, Julia Sawatzki ${ }^{53}$, Fatma Sawy $^{61}$, Michaela Schever ${ }^{50,48}$, Jacky Schuler ${ }^{45}$, Cédric Schwab ${ }^{45}$, Konstantin Schweizer ${ }^{53}$, Alexandr Selyunin ${ }^{67}$, Andrea Serafini ${ }^{56}$, Giulio Settanta ${ }^{50,64}$, Mariangela Settimo ${ }^{47}$, Zhuang Shao ${ }^{35}$, Vladislav Sharov ${ }^{67}$, Arina Shaydurova ${ }^{67}$, Jingyan $\mathrm{Shi}^{10}$, Yanan Shi ${ }^{10}$, Vitaly Shutov ${ }^{67}$, Andrey Sidorenkov ${ }^{68}$, Fedor Šimkovic ${ }^{70}$, Chiara Sirignano ${ }^{61}$, Jaruchit Siripak ${ }^{73}$, Monica Sisti ${ }^{58}$, Maciej Slupecki ${ }^{42}$, Mikhail Smirnov ${ }^{20}$, Oleg Smirnov ${ }^{67}$, Thiago Sogo-Bezerra ${ }^{47}$, Sergey Sokolov ${ }^{67}$, Julanan Songwadhana ${ }^{73}$, Boonrucksar Soonthornthum ${ }^{72}$, Albert Sotnikov ${ }^{67}$, Ondřej Šrámek ${ }^{41}$, Warintorn Sreethawong ${ }^{73}$, Achim Stahl $^{48}$, Luca Stanco ${ }^{60}$, Konstantin Stankevich ${ }^{69}$, Dušan Štefánik ${ }^{70}$, Hans Steiger ${ }^{52,53}$, Jochen Steinmann ${ }^{48}$, Tobias Sterr ${ }^{54}$, Matthias Raphael Stock ${ }^{53}$, Virginia Strati ${ }^{56}$, Alexander Studenikin ${ }^{69}$, Gongxing Sun ${ }^{10}$, Shifeng Sun ${ }^{11}$, Xilei Sun ${ }^{10}$, Yongjie Sun $^{22}$, Yongzhao Sun ${ }^{10}$, Narumon Suwonjandee ${ }^{71}$, Michal Szelezniak ${ }^{45}$, Jian Tang ${ }^{20}$, Qiang Tang ${ }^{20}$, Quan Tang ${ }^{23}$, Xiao Tang $^{10}$, Alexander Tietzsch ${ }^{54}$, Igor Tkachev ${ }^{68}$, Tomas Tmej ${ }^{41}$, Konstantin Treskov ${ }^{67}$, Andrea Triossi ${ }^{45}$, Giancarlo Troni ${ }^{5}$, Wladyslaw Trzaska ${ }^{42}$, Cristina Tuve ${ }^{55}$, Nikita Ushakov ${ }^{68}$, Johannes van den Boom ${ }^{51}$, Stefan van Waasen ${ }^{51}$, Guillaume Vanroyen $^{47}$, Nikolaos Vassilopoulos ${ }^{10}$, Vadim Vedin ${ }^{65}$, Giuseppe Verde ${ }^{55}$, Maxim Vialkov ${ }^{69}$, Benoit Viaud ${ }^{47}$, Moritz Cornelius Vollbrecht ${ }^{50,48}$, Cristina Volpe ${ }^{43}$, Vit Vorobel ${ }^{41}$, Dmitriy Voronin ${ }^{68}$, Lucia Votano ${ }^{63}$, Pablo Walker ${ }^{5}$, Caishen Wang ${ }^{18}$, Chung-Hsiang Wang ${ }^{39}$, En Wang ${ }^{37}$, Guoli Wang ${ }^{21}$, Jian Wang ${ }^{22}$, Jun Wang ${ }^{20}$, Kunyu Wang ${ }^{10}$, Lu Wang ${ }^{10}$, Meifen Wang ${ }^{10}$, Meng Wang ${ }^{23}$, Meng Wang ${ }^{25}$, Ruiguang Wang ${ }^{10}$, Siguang Wang ${ }^{12}$, Wei Wang ${ }^{27}$, Wei Wang ${ }^{20}$, Wenshuai Wang ${ }^{10}$, Xi Wang ${ }^{16}$, Xiangyue Wang ${ }^{20}$, Yangfu Wang ${ }^{10}$, Yaoguang Wang ${ }^{10}$, Yi Wang ${ }^{13}$, Yi Wang ${ }^{24}$, Yifang Wang ${ }^{10}$, Yuanqing Wang ${ }^{13}$, Yuman Wang $^{27}$, Zhe Wang ${ }^{13}$, Zheng Wang ${ }^{10}$, Zhimin Wang ${ }^{10}$, Zongyi Wang ${ }^{13}$, Muhammad Waqas ${ }^{66}$, Apimook Watcharangkool ${ }^{72}$, Lianghong Wei ${ }^{10}$, Wei Wei ${ }^{10}$, Wenlu Wei ${ }^{10}$, Yadong Wei ${ }^{18}$, Liangjian Wen ${ }^{10,}{ }^{*}{ }^{\text {(D) }}{ }^{\text {a }}$, Christopher Wiebusch ${ }^{48}$, Steven ChanFai Wong ${ }^{20}$, Bjoern Wonsak ${ }^{49}$, Diru Wu ${ }^{10}$, Fangliang $\mathrm{Wu}^{27}$, Qun Wu ${ }^{25}$, Wenjie Wu ${ }^{34}$, Zhi Wu ${ }^{10}$, Michael Wurm ${ }^{52}$, Jacques Wurtz $^{45}$, Christian Wysotzki ${ }^{48}$, Yufei Xi ${ }^{32}$, Dongmei Xia ${ }^{17}$, Yuguang Xie ${ }^{10}$, Zhangquan Xie ${ }^{10}$, Zhizhong Xing ${ }^{10}$, Benda Xu $^{13}$, Cheng $\mathrm{Xu}^{23}$, Donglian $\mathrm{Xu}^{31,30}$, Fanrong $\mathrm{Xu}^{19}$, Hangkun $\mathrm{Xu}^{10}$, Jilei $\mathrm{Xu}^{10}$, Jing $\mathrm{Xu}^{8}$, Meihang $\mathrm{Xu}^{10}$, Yin $\mathrm{Xu}^{33}$, Yu $\mathrm{Xu}^{50,48}$, Baojun Yan ${ }^{10}$, Taylor Yan ${ }^{73}$, Wenqi Yan ${ }^{10}$, Xiongbo Yan ${ }^{10}$, Yupeng Yan ${ }^{73}$, Anbo Yang ${ }^{10}$, Changgen Yang ${ }^{10}$, Huan Yang ${ }^{10}$, Jie 


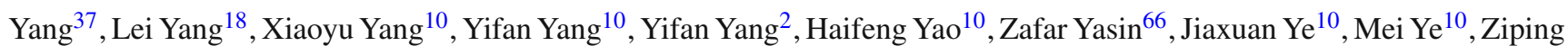
$\mathrm{Ye}^{31}$, Ugur Yegin ${ }^{51}$, Frédéric Yermia ${ }^{47}$, Peihuai $\mathrm{Yi}^{10}, \mathrm{Na} \mathrm{Yin}^{25}$, Xiangwei Yin ${ }^{10}$, Zhengyun $\mathrm{You}^{20}$, Boxiang Yu ${ }^{10}, \mathrm{Chiye} \mathrm{Yu}^{18}$, Chunxu Yu ${ }^{33}$, Hongzhao $\mathrm{Yu}^{20}$, Miao $\mathrm{Yu}^{34}$, Xianghui $\mathrm{Yu}^{33}$, Zeyuan $\mathrm{Yu}^{10}$, Zezhong Yu${ }^{10}$, Chengzhuo Yuan ${ }^{10}$, Ying Yuan ${ }^{12}$, Zhenxiong Yuan ${ }^{13}$, Ziyi Yuan ${ }^{34}$, Baobiao Yue ${ }^{20}$, Noman Zafar ${ }^{66}$, Andre Zambanini ${ }^{51}$, Vitalii Zavadskyi ${ }^{67}$, Shan Zeng ${ }^{10}$, Tingxuan Zeng ${ }^{10}$, Yuda Zeng ${ }^{20}$, Liang Zhan ${ }^{10}$, Aiqiang Zhang ${ }^{13}$, Feiyang Zhang ${ }^{30}$, Guoqing Zhang ${ }^{10}$, Haiqiong Zhang ${ }^{10}$, Honghao Zhang ${ }^{20}$, Jiawen Zhang ${ }^{10}$, Jie Zhang ${ }^{10}$, Jingbo Zhang ${ }^{21}$, Jinnan Zhang ${ }^{10}$, Peng Zhang ${ }^{10}$, Qingmin Zhang ${ }^{35}$, Shiqi Zhang $^{20}$, Shu Zhang ${ }^{20}$, Tao Zhang ${ }^{30}$, Xiaomei Zhang ${ }^{10}$, Xuantong Zhang ${ }^{10}$, Xueyao Zhang ${ }^{25}$, Yan Zhang ${ }^{10}$, Yinhong Zhang ${ }^{10}$, Yiyu Zhang ${ }^{10}$, Yongpeng Zhang ${ }^{10}$, Yuanyuan Zhang ${ }^{30}$, Yumei Zhang ${ }^{20}$, Zhenyu Zhang ${ }^{34}$, Zhijian Zhang ${ }^{18}$, Fengyi Zhao ${ }^{26}$, Jie Zhao ${ }^{10}$, Rong Zhao ${ }^{20}$, Shujun Zhao ${ }^{37}$, Tianchi Zhao ${ }^{10}$, Dongqin Zheng ${ }^{19}$, Hua Zheng ${ }^{18}$, Minshan Zheng ${ }^{9}$, Yangheng Zheng $^{14}$, Weirong Zhong ${ }^{19}$, Jing Zhou ${ }^{9}$, Li Zhou ${ }^{10}$, Nan Zhou ${ }^{22}$, Shun Zhou ${ }^{10}$, Tong Zhou ${ }^{10}$, Xiang Zhou ${ }^{34}$, Jiang Zhu ${ }^{20}$, Kejun Zhu ${ }^{10}$, Zhihang Zhu ${ }^{10}$, Bo Zhuang ${ }^{10}$, Honglin Zhuang ${ }^{10}$, Liang Zong ${ }^{13}$, Jiaheng Zou ${ }^{10}$

${ }^{1}$ Yerevan Physics Institute, Yerevan, Armenia

${ }^{2}$ Université Libre de Bruxelles, Brussels, Belgium

${ }^{3}$ Universidade Estadual de Londrina, Londrina, Brazil

${ }^{4}$ Pontificia Universidade Catolica do Rio de Janeiro, Rio, Brazil

${ }^{5}$ Pontificia Universidad Católica de Chile, Santiago, Chile

${ }^{6}$ Universidad Tecnica Federico Santa Maria, Valparaiso, Chile

${ }^{7}$ Beijing Institute of Spacecraft Environment Engineering, Beijing, China

${ }^{8}$ Beijing Normal University, Beijing, China

${ }^{9}$ China Institute of Atomic Energy, Beijing, China

${ }^{10}$ Institute of High Energy Physics, Beijing, China

${ }^{11}$ North China Electric Power University, Beijing, China

${ }^{12}$ School of Physics, Peking University, Beijing, China

${ }^{13}$ Tsinghua University, Beijing, China

${ }^{14}$ University of Chinese Academy of Sciences, Beijing, China

15 Jilin University, Changchun, China

${ }^{16}$ College of Electronic Science and Engineering, National University of Defense Technology, Changsha, China

${ }^{17}$ Chongqing University, Chongqing, China

${ }^{18}$ Dongguan University of Technology, Dongguan, China

${ }^{19}$ Jinan University, Guangzhou, China

${ }^{20}$ Sun Yat-Sen University, Guangzhou, China

${ }^{21}$ Harbin Institute of Technology, Harbin, China

${ }^{22}$ University of Science and Technology of China, Hefei, China

${ }^{23}$ The Radiochemistry and Nuclear Chemistry Group in University of South China, Hengyang, China

${ }^{24}$ Wuyi University, Jiangmen, China

${ }^{25}$ Shandong University, Jinan, China, and Key Laboratory of Particle Physics and Particle Irradiation of Ministry of

Education, Shandong University, Qingdao, China

${ }^{26}$ Institute of Modern Physics, Chinese Academy of Sciences, Lanzhou, China

${ }^{27}$ Nanjing University, Nanjing, China

${ }^{28}$ Guangxi University, Nanning, China

${ }^{29}$ East China University of Science and Technology, Shanghai, China

${ }^{30}$ School of Physics and Astronomy, Shanghai Jiao Tong University, Shanghai, China

${ }^{31}$ Tsung-Dao Lee Institute, Shanghai Jiao Tong University, Shanghai, China

${ }^{32}$ Institute of Hydrogeology and Environmental Geology, Chinese Academy of Geological Sciences, Shijiazhuang, China

${ }^{33}$ Nankai University, Tianjin, China

${ }^{34}$ Wuhan University, Wuhan, China

${ }^{35} \mathrm{Xi}$ ' an Jiaotong University, Xi' an, China

${ }^{36}$ Xiamen University, Xiamen, China

${ }^{37}$ School of Physics and Microelectronics, Zhengzhou University, Zhengzhou, China

${ }^{38}$ Institute of Physics, National Yang Ming Chiao Tung University, Hsinchu

${ }^{39}$ National United University, Miao-Li, Taiwan

${ }^{40}$ Department of Physics, National Taiwan University, Taipei 
${ }^{41}$ Charles University, Faculty of Mathematics and Physics, Prague, Czech Republic

${ }^{42}$ University of Jyvaskyla, Department of Physics, Jyvaskyla, Finland

${ }^{43}$ IJCLab, Université Paris-Saclay, CNRS/IN2P3, 91405 Orsay, France

${ }^{44}$ Univ. Bordeaux, CNRS, CENBG, UMR 5797, F-33170 Gradignan, France

45 IPHC, Université de Strasbourg, CNRS/IN2P3, F-67037 Strasbourg, France

${ }^{46}$ Centre de Physique des Particules de Marseille, Marseille, France

${ }^{47}$ SUBATECH, Université de Nantes, IMT Atlantique, CNRS-IN2P3, Nantes, France

${ }^{48}$ III. Physikalisches Institut B, RWTH Aachen University, Aachen, Germany

${ }^{49}$ Institute of Experimental Physics, University of Hamburg, Hamburg, Germany

${ }^{50}$ Forschungszentrum Jülich GmbH, Nuclear Physics Institute IKP-2, Jülich, Germany

${ }^{51}$ Forschungszentrum Jülich GmbH, Central Institute of Engineering, Electronics and Analytics - Electronic Systems (ZEA-2), Jülich, Germany

52 Institute of Physics and Excellence Cluster PRISMA ${ }^{+}$, Johannes-Gutenberg Universität Mainz, Mainz, Germany

53 Technische Universität München, München, Germany

${ }^{54}$ Eberhard Karls Universität Tübingen, Physikalisches Institut, Tübingen, Germany

55 INFN Catania and Dipartimento di Fisica e Astronomia dell Università di Catania, Catania, Italy

${ }^{56}$ Department of Physics and Earth Science, University of Ferrara and INFN Sezione di Ferrara, Ferrara, Italy

${ }^{57}$ INFN Sezione di Milano and Dipartimento di Fisica dell Università di Milano, Milano, Italy

${ }^{58}$ INFN Milano Bicocca and University of Milano Bicocca, Milano, Italy

${ }^{59}$ INFN Milano Bicocca and Politecnico of Milano, Milano, Italy

${ }^{60}$ INFN Sezione di Padova, Padova, Italy

${ }^{61}$ Dipartimento di Fisica e Astronomia dell'Università di Padova and INFN Sezione di Padova, Padova, Italy

62 INFN Sezione di Perugia and Dipartimento di Chimica, Biologia e Biotecnologie dell'Università di Perugia, Perugia, Italy

${ }^{63}$ Laboratori Nazionali di Frascati dell'INFN, Roma, Italy

${ }^{64}$ University of Roma Tre and INFN Sezione Roma Tre, Roma, Italy

65 Institute of Electronics and Computer Science, Riga, Latvia

${ }^{66}$ Pakistan Institute of Nuclear Science and Technology, Islamabad, Pakistan

${ }^{67}$ Joint Institute for Nuclear Research, Dubna, Russia

${ }^{68}$ Institute for Nuclear Research of the Russian Academy of Sciences, Moscow, Russia

${ }^{69}$ Lomonosov Moscow State University, Moscow, Russia

${ }^{70}$ Comenius University Bratislava, Faculty of Mathematics, Physics and Informatics, Bratislava, Slovakia

${ }^{71}$ Department of Physics, Faculty of Science, Chulalongkorn University, Bangkok, Thailand

72 National Astronomical Research Institute of Thailand, Chiang Mai, Thailand

${ }^{73}$ Suranaree University of Technology, Nakhon Ratchasima, Thailand

${ }^{74}$ Department of Physics and Astronomy, University of California, Irvine, California, USA 